1 CD4 Effectors Need to Recognize Antigen Locally to Become Cytotoxic CD4

\title{
and Follicular Helper T Cells
}

$6{ }^{1}$ Department of Pathology, University of Massachusetts Medical School, Worcester, MA 01605, 7 USA.

$8{ }^{2}$ Department of Animal Medicine, University of Massachusetts Medical School, Worcester, MA 901605 , USA.

10 \# P.D. and A.M.V. contributed equally to this work.

11 *Corresponding author: Susan L. Swain, Department of Pathology, University of Massachusetts

12 Medical School, Worcester, MA 01605, USA.

13 Phone: 508-856-4494 (office) Email id: $\underline{\text { susan.swain@umassmed.edu }}$ 


\section{Summary}

$24 \mathrm{~T}$ follicular helper $\left(\mathrm{T}_{\mathrm{FH}}\right)$ and Cytotoxic CD4 (ThCTL) are tissue-restricted CD4 effector subsets,

25 functionally specialized to mediate optimal $\mathrm{Ab}$ production and cytotoxicity of infected cells.

26 Influenza infection generates robust CD4 responses, including lung ThCTL and SLO TFH, that

27 protect against reinfection by variant strains. Antigen (Ag) presentation after infection, lasts

28 through the effector phase of the response. Here, we show that this effector phase Ag presentation,

29 well after priming, is required to drive CD4 effectors to ThCTL and $\mathrm{T}_{\mathrm{FH}}$. Using in vivo influenza

30 models, we varied Ag presentation to effectors acutely, just at the effector phase. Ag presentation

31 was required in the tissue of effector residence. We suggest these requirements contain

32 unnecessary or potentially pathogenic CD4 responses, only allowing them if infection is uncleared.

33 The results imply that providing effector phase Ag, would lead to stronger humoral and CD4 tissue

34 immunity and thus can be applied to improve vaccine design.

37 Keywords: Tissue-Restricted, Effectors, Influenza, Pathogen, Vaccination, CD4 T cells,

38 Immunization, $\mathrm{T}$ Cytotoxic, $\mathrm{T}$ Helper, $\mathrm{T}_{\mathrm{FH}}$ 


\section{INTRODUCTION}

45 A key challenge for the immune system is to respond strongly against dangerous pathogens, while

46 limiting response to non-threatening foreign antigens, so as to limit immunopathology. Naïve CD4

47 T cells achieve this discrimination by requiring three signals during priming at the beginning of the response to generate the initial effector populations: high levels of antigen (Ag), co-stimulation and inflammatory cytokines (Dubey and Croft, 1996). These effectors defend the body against foreign Ag during the effector phase and finally contract after the Ag has waned to become long lived memory T cells. A cohort of the CD4 effectors can differentiate further, to become tissuerestricted effectors, including $\mathrm{T}$ follicular helpers $\left(\mathrm{T}_{\mathrm{FH}}\right)$ (Fazilleau et al., 2009; Lee et al., 2015) and cytotoxic CD4 T cells (ThCTL) (Marshall et al., 2016).

$54 \mathrm{~T}_{\mathrm{FH}}$ are tissue-restricted CD4 effectors in the secondary lymphoid organs such as the spleen and lymph nodes (Fazilleau et al., 2009; Lee et al., 2015). They drive the germinal center reaction and

56 the resulting antibody (Ab) responses during the immune response (Crotty, 2019). ThCTL are

57 cytotoxic CD4 effectors that target cells expressing MHC-II, which may also have downregulated

58 MHC-I due to evasion mechanisms, and play important roles in controlling viral infections and tumors (Juno et al., 2017; Koutsakos et al., 2019a; Marshall and Swain, 2011; Marshall et al., 2016; Muraro et al., 2017; Phetsouphanh et al., 2017). Previously, we showed that ThCTL induced by viral infections are resident in the infected tissue (Marshall et al., 2016). During influenza virus infection, ThCTL arise 7 days post infection (dpi) in lungs of infected mice. ThCTL express

$63 \mathrm{NKG} 2 \mathrm{C} / \mathrm{E}$, which reliably marks the CD4 effector subset with MHC-II restricted cytotoxicity. 
and cancer, little is known about the signals that regulate the transition of early CD4 effectors into these later tissue effectors.

Our current understanding of CD4 tissue-restricted effectors is largely inferred from studies of $\mathrm{T}_{\mathrm{FH}}$ during $\mathrm{T}: \mathrm{B}$ interaction in responding germinal centers. Over the past decade we have learned that after priming, $\mathrm{T}_{\mathrm{FH}}$ repeatedly interact with B cells in germinal centers to support GC responses.

71 The germinal center $\mathrm{B}$ cells $(\mathrm{GCB})$ reciprocally support $\mathrm{T}_{\mathrm{FH}}$ differentiation, survival and expansion

72 (Vinuesa et al., 2016). Studies have shown that $\mathrm{T}_{\mathrm{FH}}$ generation is enhanced by repeated 73 immunization (Baumjohann et al., 2013; Deenick et al., 2010; Tam et al., 2016), but it is unclear

74 if $\mathrm{Ag}$ is required both for initial priming and at the effector phase. CD4 effectors generated during LCMV infection, depended on ongoing infection to effectively generate $\mathrm{T}_{\mathrm{FH}}$ (Baumjohann et al., 2013). In this study it was unclear whether Ag, or infection-generated inflammation, or both, were required to support $\mathrm{T}_{\mathrm{FH}}$ generation during the effector phase and if $\mathrm{Ag}$ presentation needed to occur in particular locations. While we know that $\mathrm{T}_{\mathrm{FH}}$ continuously interact with GCB that present $\mathrm{Ag}$ in situ, we do not know which aspects of this interaction are required during the effector phase, to regulate the development of CD4 tissue effector subsets. Moreover, we do not know if the GC presents a niche, unique in its ability to fulfill requirements for CD4 tissue effector differentiation or if there are other signals that are able to substitute for them. An immunization study indicated that GCB depletion during the effector phase reduced $\mathrm{T}_{\mathrm{FH}}$ generation (Baumjohann et al., 2013). Another study showed that early $\mathrm{T}_{\mathrm{FH}}$ generation can occur independently of unique $\mathrm{B}$ cell signaling

85 (Deenick et al., 2011), supporting the concept that when other APC presenting Ag are available, $\mathrm{T}_{\mathrm{FH}}$ may be able to develop by GCB independent pathways even during the effector phase, though

87 this is unknown. Moreover, other CD4 tissue effectors such as ThCTL are not present in GC and we have little understanding of their development from early CD4 effectors, or if they have shared 
or unique requirements with $\mathrm{T}_{\mathrm{FH}}$. Thus, while the $\mathrm{GC}$ studies give us many important clues about CD4 tissue effectors, they leave many questions unanswered. To fill these gaps, here we analyze

91 the overall requirements for different aspects of Ag encounter, specifically during the CD4 effector

92 phase well after priming, that drive CD4 effectors to become tissue-restricted effectors.

93 We previously showed that CD4 effectors generated by influenza virus infection need to recognize

94 Ag during the effector phase, 6-8 dpi, to effectively form long-lived memory (Bautista et al., 2016; McKinstry et al., 2014). Here, we ask if late steps in generation of $\mathrm{T}_{\mathrm{FH}}$ and ThCTL tissue effector

96 subsets, also require cognate Ag recognition at this "effector checkpoint". We reason that if an

97 infection is quickly cleared or initial Ag is non-replicating, presentation of $\mathrm{Ag}$ will wane. Thus, 98 such a checkpoint could act to limit further response when the infection (the source of $\mathrm{Ag}$ ) is 99 cleared, serving as a mechanism to prevent immunopathology and potential autoimmunity when 100 there is no longer danger from a live pathogen.

101 Here, we find that CD4 effectors must recognize cognate Ag during the effector checkpoint to 102 become full-fledged ThCTL and $\mathrm{T}_{\mathrm{FH}}$, and that multiple APC can support this transition. Moreover, 103 for full development of $\mathrm{DLN} \mathrm{T}_{\mathrm{FH}}$, spleen $\mathrm{T}_{\mathrm{FH}}$ and lung $\mathrm{ThCTL}$, effectors must recognize Ag 104 presented at the site of tissue effector residency. CD28 co-stimulation during the effector 105 checkpoint is required for $\mathrm{T}_{\mathrm{FH}}$, but not for ThCTL generation. Thus, at the effector phase, well 106 after Ag priming, multiple signals during cognate Ag recognition act in concert to drive different 107 specialized CD4 fates: ThCTL, $\mathrm{T}_{\mathrm{FH}}$ and CD4 memory. This suggests that this effector CD4 108 checkpoint regulates the quality, quantity and localization of CD4 tissue-restricted effectors and 109 the memory cells they become. We discuss the relevance of these findings to designing vaccine 
strategies that could induce effective long-lived $\mathrm{Ab}$ and cellular immunity against conserved epitopes.

\section{RESULTS}

\section{Cognate Ag recognition at the CD4 effector checkpoint drives the generation of ThCTL}

\section{phenotype and function in the lung}

We use a sequential transfer model, in which 6 dpi CD4 effectors are generated in vivo by transferring naïve HNT (specific for influenza A virus hemagglutinin) or OT-II (specific for an OVA epitope) Thy1.1 CD4 T cells, into primary hosts infected with PR8 or with PR8-OVA influenza virus respectively (for 6 days). We then isolate the in vivo generated effectors and transfer them into $2^{\text {nd }}$ hosts. The $2^{\text {nd }}$ hosts are IAV infection-matched mice, i.e. also at 6 dpi when 6 dpi effectors are transferred into them, to make the model physiologically relevant. In the $2^{\text {nd }}$ hosts, we can independently modulate Ag availability by using Ag-pulsed APC (Ag/APC) transfers or virus infections. This allows us to clearly follow donor cell fate to ask specific questions about cognate $\mathrm{Ag} / \mathrm{APC}$ interactions during the effector phase.

In all experiments using the sequential transfer model, we analyze the transferred donor effector cells 2-4 days post transfer (8-10 days post infection) and not later, because ThCTL and $\mathrm{T}_{\mathrm{FH}}$ peak 7-10 days post infection (Marshall et al., 2016) (Fig S1D-E), and then effectors begin to contract after 10 dpi (Botta et al., 2017; Marshall et al., 2016). Signals required for CD4 T cell priming during the first few days are well-defined (Swain et al., 2012). The sequential transfer model allows us to define signals required during the effector phase, well after priming, but before contraction. Since there is robust generation of both ThCTL and $\mathrm{T}_{\mathrm{FH}}$ during an in vivo influenza infection (Figure S1D-E), they serve as positive controls. Thus an important advantage of this TCR Tg 

$\left.\begin{array}{l}\text { PR8-OVA infection matched (both influenza infection and antigen available) } \\ \text { PR8 infection matched (influenza infection available but no antigen) } \\ \text { Uninfected (no influenza infection and no antigen) }\end{array}\right] \begin{gathered}\text { 8d } \\ \text { Harvest } \\ \text { Analyse } \\ \text { ThCTL (Fig 1) } \\ T_{F H} \text { (Fig 2) }\end{gathered}$

B

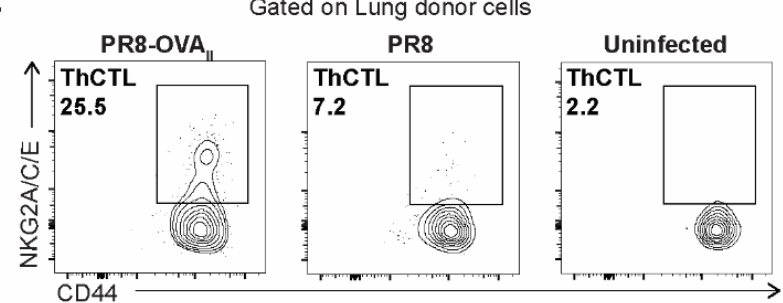

ThCTL
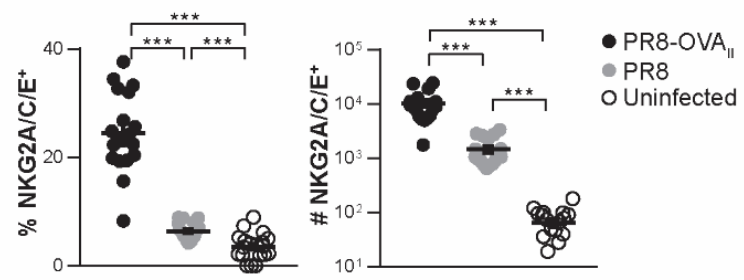

C

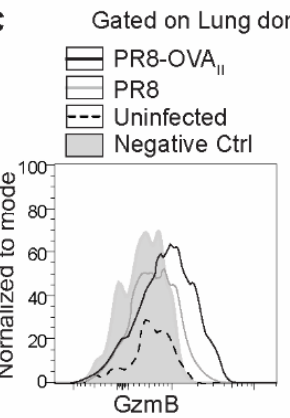

E

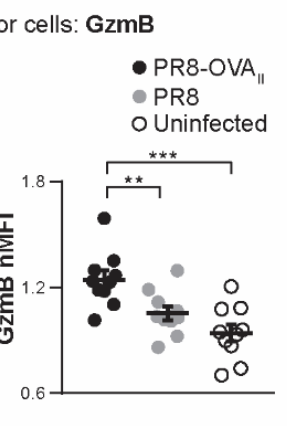

D
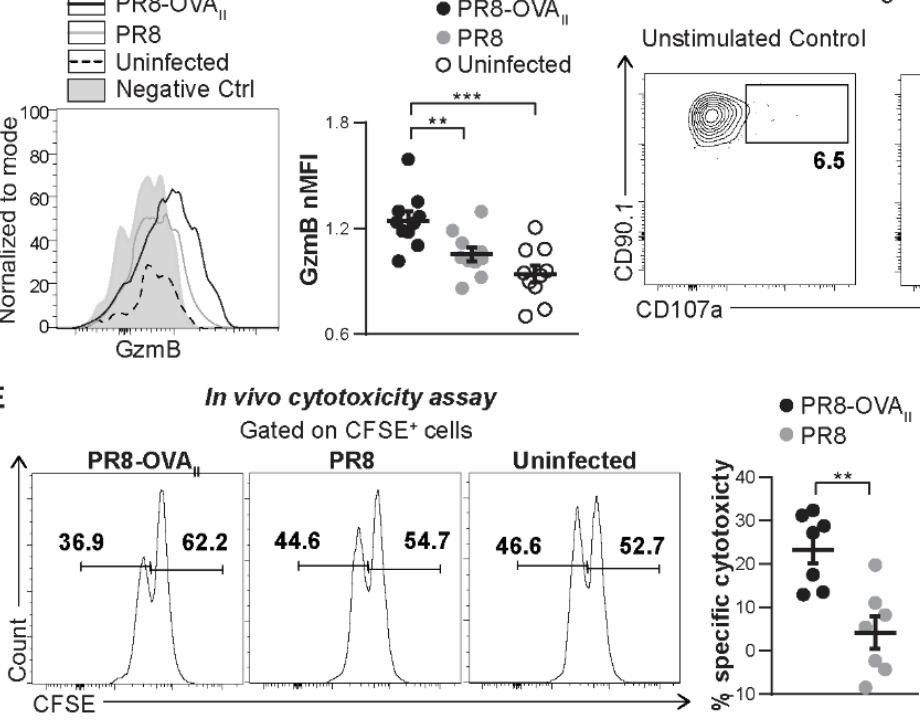

Gated on Lung donor cells: CD107a

PR8-OVA

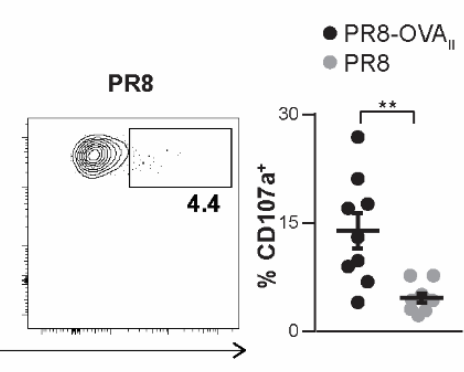

F

G
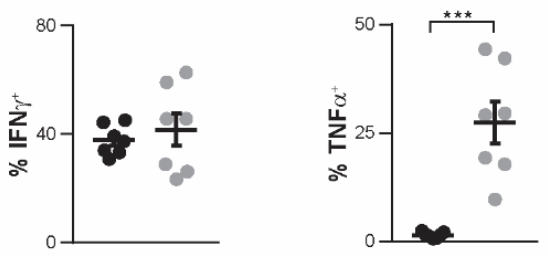

Fig. 1. Cognate Ag during the effector checkpoint is required for lung ThCTL phenotype and function. (A) Experimental design for (B-D): Naïve OT-II.Thy $1.1^{+}$cells were transferred into PR8-OVA infected mice $\left(1^{\text {st }}\right.$ hosts). At 6 dpi, OT-II.Thy $1.1^{+}$effectors were isolated from $1^{\text {st }}$ hosts and transferred into following groups of $2^{\text {nd }}$

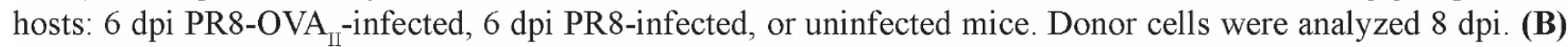
Percentage and numbers of donor lung ThCTL $\left(\mathrm{NKG} 2 \mathrm{~A} / \mathrm{C} / \mathrm{E}^{+}\right)(\mathrm{n}=19$ per group pooled, 4 independent experiments). (C) Representative histogram of lung donor cell GzmB expression (negative control: naïve CD4 from uninfected mice). Normalized MFI of lung donor cell GzmB expression ( $\mathrm{n}=10$ per group pooled, 2 independent experiments). (D) CD107a degranulation marker expression by lung donor cells ( $\mathrm{n}=9$ per group pooled, 2 independent experiments). (E) Experimental design: In vivo 6d OT-II.Thy $1.1^{+}$effectors were transferred into 6 dpi PR8-OVA or PR8 infection-matched TCR $\alpha / \beta^{-/}$mice. $\mathrm{CFSE}^{\text {lo }}$ target and $\mathrm{CFSE}^{\text {hi }}$ bystander cells were transferred at 7d. Representative CFSE histograms shown. Percentage Ag specific cytotoxicity in each group is shown. (F-G) Experiment done as in (E). Percentage of lung donor cells expressing intracellular IFN $\gamma(\mathrm{F})$ and TNF $\alpha(\mathrm{G})$ (E-G, $n=7$ per group pooled, 2 independent experiments). Statistical significance determined by two-tailed, unpaired Student's t-test $\quad(* \quad \mathrm{P}<0.05, \quad * * \quad \mathrm{P}<0.01, \quad * * * \quad \mathrm{P}<0.001) . \quad$ See also $\quad$ Fig. $\quad$ S2. 
151 We first analyzed if early ThCTL had recently recognized Ag during an in vivo influenza infection.

152 For this, we used influenza-specific TCR transgenic CD4 T (OT-II specific for PR8-OVA II) mice

153 crossed to the Nur77 $7^{\mathrm{GFP}}$ reporter mice, as a source of reporter CD4 T cells, to track recent TCR

154 stimulation. Nur77 $7^{\mathrm{GFP}}$ cells transiently express $\mathrm{Nur} 77^{\mathrm{GFP}}$ when they are stimulated by Ag

155 recognition (Au-Yeung et al., 2014; Bautista et al., 2016; Moran et al., 2011). Naïve OT-

156 II.Nur77 ${ }^{\mathrm{GFP}}$.Thy $1.1^{+}$CD4 T cells were transferred into wild-type (WT) hosts infected with PR8-

157 OVA

158 higher in the Nur7 $7^{\text {GFP+ }}$ subset at 6 dpi compared to the Nur77 ${ }^{\text {GFP- }}$ subset (Fig S1A), indicating

159 they have recently recognized $\mathrm{Ag}$.

160 We used the sequential transfer approach (Bautista et al., 2016) to determine if 6 dpi effectors

161 require Ag to become ThCTL (Figure 1A). We transferred naïve OT-II cells into hosts that were

162 then infected with PR8-OVA (II $^{\text {st }}$ hosts). We isolated in vivo-generated OT-II effectors at 6 dpi.

163 These were transferred into $2^{\text {nd }}$ hosts that had been infected $6 \mathrm{~d}$ previously (infection-matched).

164 The $2^{\text {nd }}$ hosts provided either Ag and infection (PR8-OVAII infection), infection without Ag (PR8

165 infection) or neither (uninfected) (Figure 1A). Donor ThCTL generation was assessed 2 days post

166 transfer (dpt) in the lung which corresponded to 8 days post infection. Donor ThCTL developed

167 when $\mathrm{Ag}$ was presented in the $2^{\text {nd }}$ hosts as expected (positive control), but their number was

168 drastically reduced when Ag was absent (7x) (Figure 1B). The Ag-dependence was selective for

169 ThCTL, as the total number of donor lung effectors was only reduced 1.7x (Fig S2A). Donor

170 expression of indicators of cytotoxicity, that also characterize ThCTL, such as Granzyme B

171 (GzmB) (Figure 1C) and the degranulation marker CD107a (Figure 1D), also depended on Ag

172 presented in the $2^{\text {nd }}$ host. Expression of other ThCTL-associated markers (Marshall et al., 2016) 
173 by the donor lung effectors: PD1, CXCR6 and SLAM, were also Ag dependent, but active PSGL1

174 and CXCR3 were not (Figure S2B-F).

175 To assess in vivo cytotoxic function, the in vivo-generated effectors were transferred into $2^{\text {nd }}$ hosts

176 with or without Ag (as in Figure 1A) and additionally with CFSE-labeled target cells (Figure 1E).

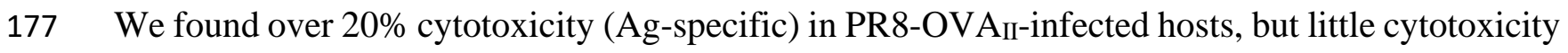

178 in PR8-infected hosts. Thus, donor cell mediated CD4 cytotoxicity only developed when effectors

179 were exposed to Ag, correlating with percentage and number of ThCTL in Figure 1B.

We also analyzed secretion of canonical Th1 effector cytokines such as IFN $\gamma$ and TNF $\alpha$ by donor cells. In contrast to ThCTL that depend on $\mathrm{Ag}$, the donor effectors recovered did not require $\mathrm{Ag}$ recognition to maintain the ability to secrete IFN $\gamma$ and in fact $\mathrm{TNF} \alpha$ secretion was lost when cognate Ag was present during the effector checkpoint (Figure 1F-G, Figure S2G). Thus, a

184 program leading to induction of ThCTL phenotypes and functions, but not general Th1 characteristics, were coordinately driven in 6d effectors by cognate Ag recognition.

Cognate Ag recognition at the effector checkpoint drives generation of $\mathbf{T}_{\mathrm{FH}}, \mathrm{GC}-\mathrm{T}_{\mathrm{FH}}$ and

$188 \mathrm{~T}_{\mathrm{FH}}$, like ThCTL, peak at 7-8 dpi (Figure S1D) and express specialized tissue-restricted and 189 functional programs (Fazilleau et al., 2009; Lee et al., 2015; Vinuesa et al., 2016). In the same 190 experiment as in Figure $\mathrm{S} 1 \mathrm{~A}, \mathrm{~T}_{\mathrm{FH}}\left(\mathrm{CXCR} 5^{\mathrm{hi}} \mathrm{Bcl6}^{\mathrm{hi}}\right)$ were also enriched in the Nur7 $7^{\mathrm{GFP}+}$ population 191 at 6 dpi in both the lung draining lymph nodes (DLN) and the spleen, indicating they had recently 192 recognized Ag (Figure S1B-C). To test if 6d effectors require cognate Ag recognition to fully 193 develop into $\mathrm{T}_{\mathrm{FH}}$ in the secondary lymphoid organs (SLO), we assessed $\mathrm{T}_{\mathrm{FH}}$ and more 194 differentiated germinal center $\mathrm{T}_{\mathrm{FH}}\left(\mathrm{GC}-\mathrm{T}_{\mathrm{FH}}\right)$ using the sequential transfer system described in 195 Figure 1A. In the PR8-OVA $\mathrm{II}$ infection-matched positive controls, a strong donor $\mathrm{T}_{\mathrm{FH}}$ response 
197 found in either the spleen (Figure 2A) or the DLN (Figure S3B).

A

Gated on Spleen donor cells
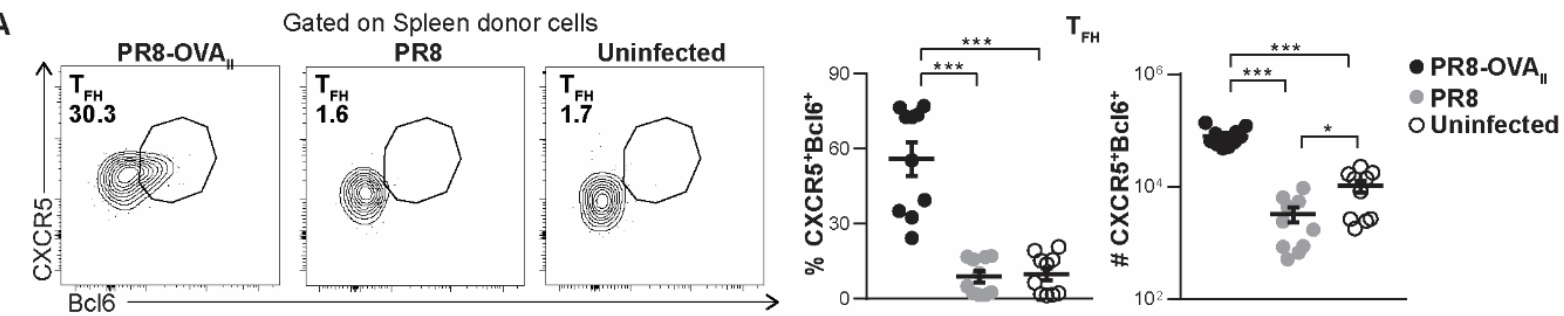

200

B Gated on Spleen donor $\mathrm{T}$
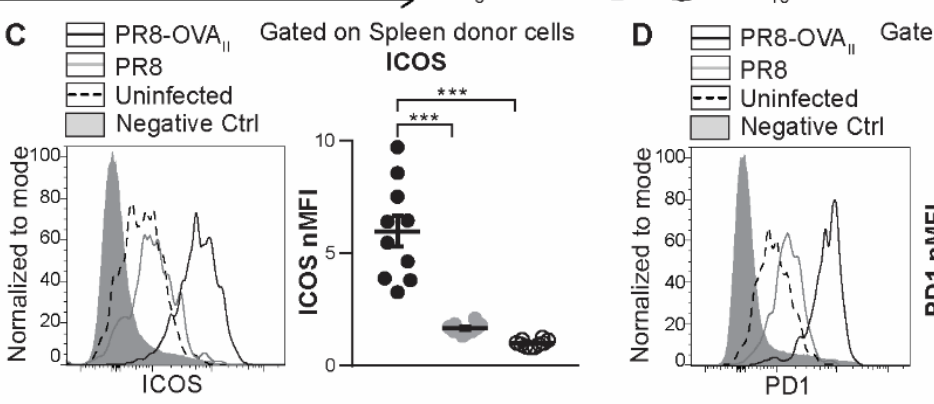

ated on Spleen donor cells

201 GC-T $\mathrm{FH}_{\mathrm{FH}}$

202

203

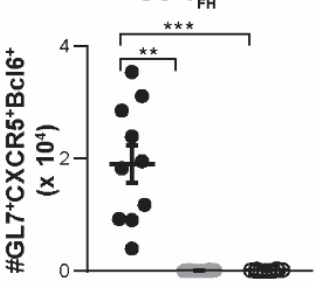

Gated on total Spleen donor cells: IL-21, IFN $\gamma$, TNF $\alpha$ cytokine secretion

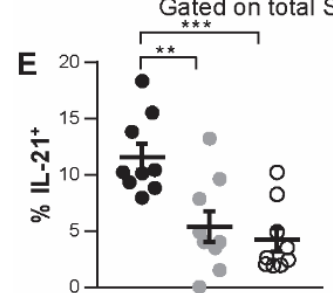

206

$\mathrm{H}$

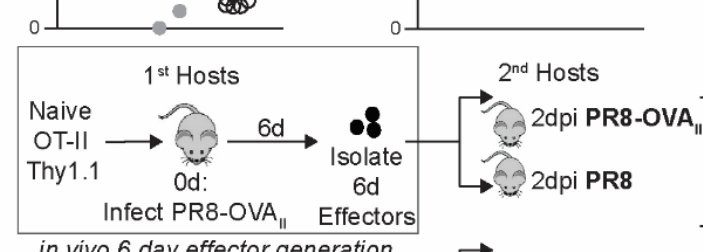

207

208

209

J Gated on host Spleen CD19+Fas ${ }^{+} \mathrm{GL7} 7^{+} \mathrm{BCl6}^{+} \mathrm{GCB}$ cells

Negative Control: No transfer-
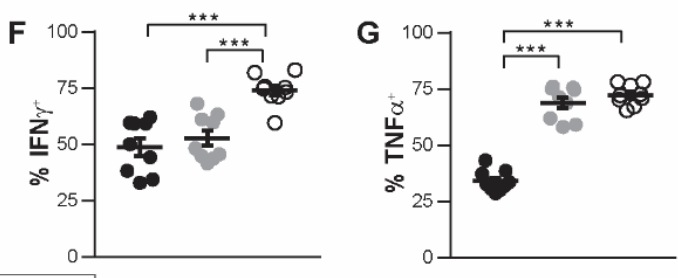

- PR8-OVA

- PR8

PD1

OUninfected
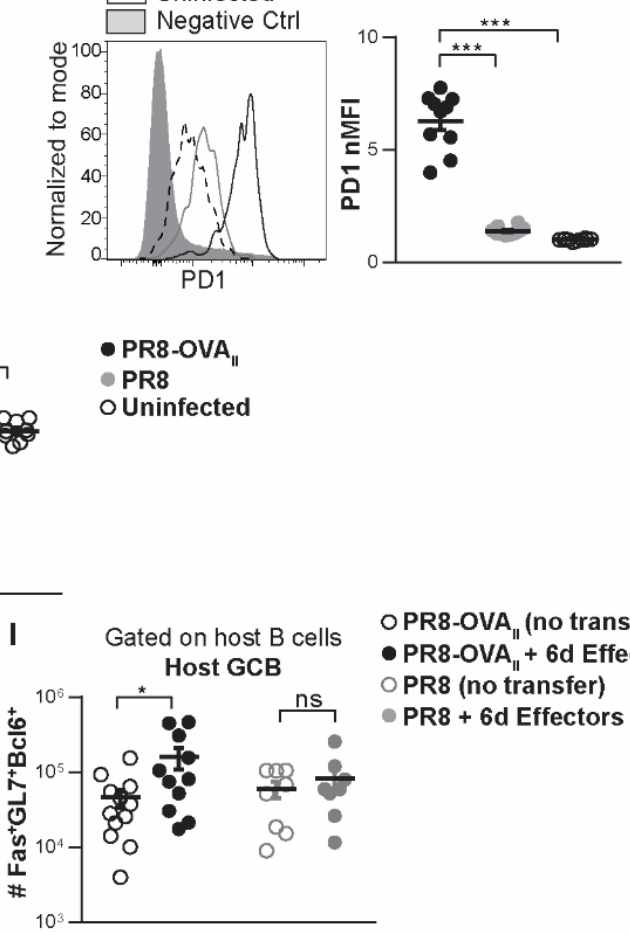

OPR8-OVA ${ }^{11}$ (no transfer) - PR8-OVA + + 6d Effectors O PR8 (no transfer) - PR8 + 6d Effectors

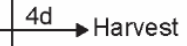
Host GCB formation
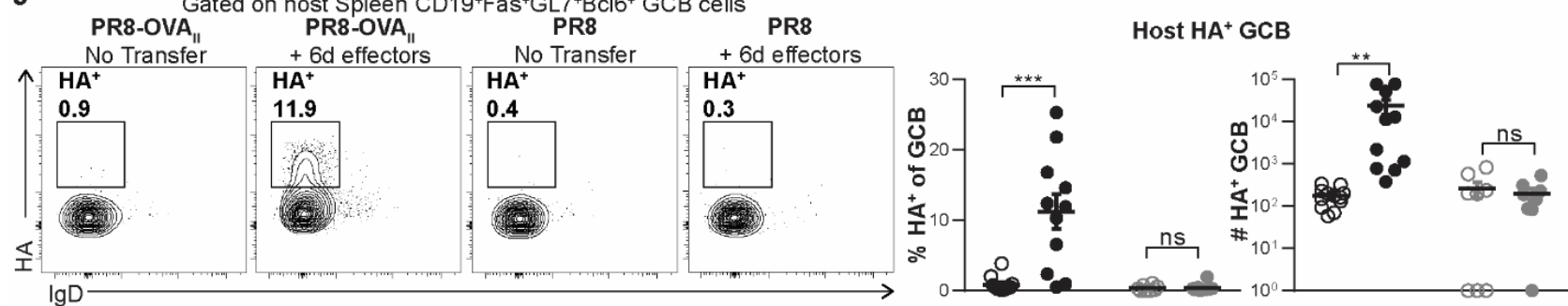

Fig. 2. SLO $\mathrm{T}_{\mathrm{FH}}$ require Ag recognition during the effector checkpoint. Experiment performed as in Fig. 1A

for Fig. 2A-G. (A) Percentage and numbers of spleen donor $\mathrm{T}_{\mathrm{FH}}\left(\mathrm{CXCR} 5^{+} \mathrm{Bcl}^{+}\right.$). (B) Number of spleen donor germinal center $\mathrm{T}_{\mathrm{FH}}\left(\mathrm{GL}^{+} \mathrm{CXCR}^{+} \mathrm{Bcl}^{+}\right)$. (C-D) Representative histogram of ICOS (C) and PD1 (D) expression by spleen donor cells (negative control: naïve CD4 from uninfected mice). Normalized ICOS MFI (C) and PD1 MFI (D) expression by spleen donor cells. (A-D, n= 10 per group pooled, 2 independent experiments). (E-G) Percentage of spleen donor cells expressing intracellular IL-21 (E), IFN $\gamma(\mathrm{F})$ and TNF $\alpha(\mathrm{G})$ ( $\mathrm{n}=9$ per

group pooled, 2 independent experiments). (H) Experimental design for (I-J): In vivo generated $6 \mathrm{~d}$ OT-II.Thy $1.1^{+}$effectors were transferred into 2 dpi PR8-OVA II $^{-}$fifected or PR8-infected mice. A group of 2 dpi

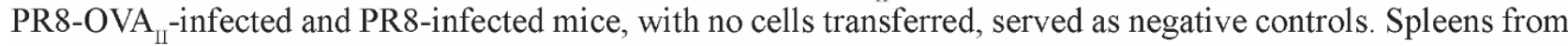
these mice were analyzed $4 \mathrm{dpt}$. (I) Number of host GCB cells (CD19 ${ }^{+}$as $^{+} \mathrm{GL}^{+} \mathrm{Bcl6}^{+}$) formed. (J) Percentage and numbers of $\mathrm{HA}^{+} \mathrm{GCB}$. (H-I, n=8-12 per group pooled, 2-3 independent experiments). Error bars represent s.e.m. Statistical significance determined by two-tailed, unpaired Student's t-test $\left({ }^{*} \mathrm{P}<0.05, * * \mathrm{P}<0.01, * * * \mathrm{P}\right.$ $<0.001)$. See also Fig. S3 
215 Moreover, while they developed well in hosts with Ag, no donor GC-T $\mathrm{TH}_{\mathrm{FH}}$ were generated without

216 Ag (Figure 2B, Figure S3C-D). $\mathrm{T}_{\mathrm{FH}} / \mathrm{GC}-\mathrm{T}_{\mathrm{FH}}$-associated molecules PD1 and ICOS were highly

217 expressed by day 8 only when Ag was available (Figure 2C-D, Figure S3E-F), and were reduced

218 in the absence of Ag. Thus, results from the same sequential transfer experiment (Figure 1A)

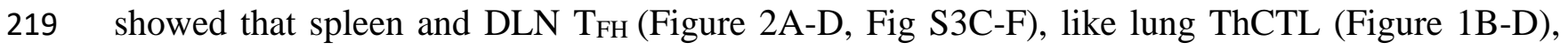

220 required cognate $\mathrm{Ag}$ recognition during the effector phase.

221 IL-21 promotes $\mathrm{T}_{\mathrm{FH}}$ differentiation and is also produced by $\mathrm{T}_{\mathrm{FH}}$. It mediates $\mathrm{T}_{\mathrm{FH}}$ function during

222 the GC response (Vinuesa et al., 2016). Indeed, the proportion of donor effectors secreting IL-21

223 was higher in $2^{\text {nd }}$ hosts with Ag than in those without Ag (Figure 2E, Figure S3G). In contrast, the

224 ability to maintain production of cytokines not directly associated with $\mathrm{T}_{\mathrm{FH}}$, such as IFN $\gamma$ and

225 TNFa were not Ag-dependent (Figure 2F-G). This indicates a selective dependence of $\mathrm{T}_{\mathrm{FH}^{-}}$

226 associated programs, but not other effector functions, on Ag recognition during the effector

227 checkpoint.

228 To evaluate the impact of Ag recognition at the effector checkpoint, on $\mathrm{T}_{\mathrm{FH}}$ function of helping

229 GCB formation, we developed an in vivo GCB assay (Figure 2H). Endogenous host GCB are

230 undetectable from 2-6 dpi after influenza infection (Figure $\mathrm{S} 3 \mathrm{H}$ ) because $\mathrm{T}_{\mathrm{FH}}$ have not yet fully

231 formed (Figure S1D). However, we reasoned that if functional $\mathrm{T}_{\mathrm{FH}}$ were available earlier, they

232 should accelerate GCB formation. Therefore, we transferred in vivo generated 6d OT-II effectors

233 into hosts infected $2 \mathrm{~d}$ previously with either PR8 (no cognate Ag) or PR8-OVA

234 available) and analyzed host GCB 4d after transfer (6 dpi). Thus, this model allowed us to study

235 the acceleration of host GCB formation, during a timeframe (2-6 dpi) when their endogenous GCB

236 formation was low. Transfer of $6 \mathrm{~d}$ donor effectors into PR8-OVA $\mathrm{II}$-infected mice, caused a

237 significant increase in total GCB formation (Figure 2I) and $\mathrm{HA}^{+} \mathrm{GCB}$ formation (Figure 2J) while 
their transfer to PR8-infected hosts did not boost GCB formation over the negative controls which received no effectors. These results indicate that the critical $\mathrm{T}_{\mathrm{FH}}$ function of inducing $\mathrm{GCB}$ cells and thus protective $\mathrm{Ab}$ responses, requires cognate $\mathrm{Ag}$ during the effector phase. Therefore,

241 effector phase recognition of $\mathrm{Ag}$ is needed to drive induction of the key defining phenotypic and

242 functional aspects of both ThCTL and $\mathrm{T}_{\mathrm{FH}}$ programs, leading to their development in their

243 respective niches.

\section{TFH but not ThCTL require CD28 co-stimulation at the effector checkpoint}

245 T cells express CD28, which interacts with CD80/86 on APC during cognate interaction, co246 stimulating IL-2 production and initiating proliferation (Watts, 2010). We analyzed whether

247 ThCTL generation requires CD28:CD80/CD86 co-stimulation during the effector checkpoint. 6d

248 in vivo effectors were transferred into WT or CD80/86 deficient PR8-OVA

249 hosts (Figure 3A). The recovery of total donor effectors in the lung was decreased (Figure S4A),

250 when hosts lacked costimulatory ligands, but the proportion of lung donor ThCTL was increased

251 (Figure 3B). Donor ThCTL numbers were unchanged (Figure 3B), suggesting non-ThCTL were

252 lost, while ThCTL were retained. The level of NKG2A/C/E expression on donor ThCTL was 253 increased in CD80/86KO compared with WT (Figure S4B) hosts, suggesting that the

254 differentiation of ThCTL improved without CD28:CD80/86 co-stimulation. Next, we cultured in 255 vivo-generated $6 \mathrm{~d}$ effectors for $2 \mathrm{~d}$ in vitro with anti-CD3 vs anti-CD3 plus CD28, to mimic effector 256 phase Ag exposure, with and without CD28 co-stimulation (Figure 3C). No ThCTL developed 257 without anti-CD3 (Figure S4C-D), mimicking the in vivo requirement for cognate Ag during the 258 effector phase, as shown in Figure 1. 


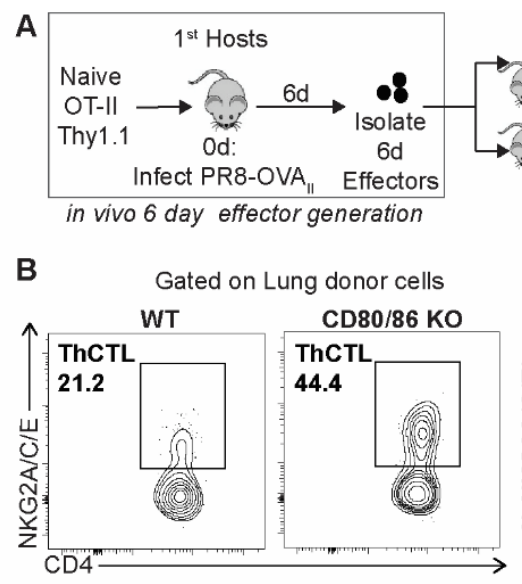
$2^{\text {nd }}$ Hosts

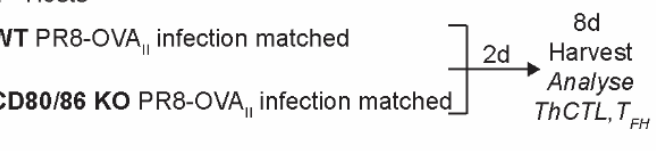

$\bullet \mathrm{WT}$

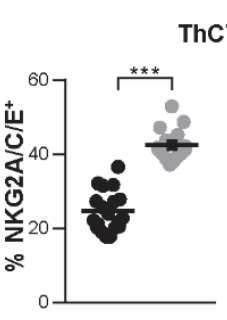

ThCTL CD80/86 KO

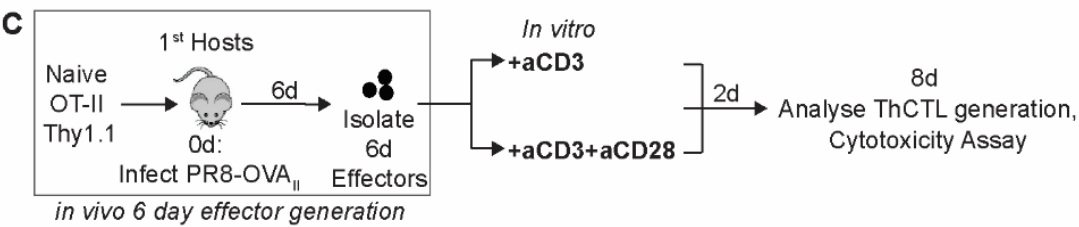

D
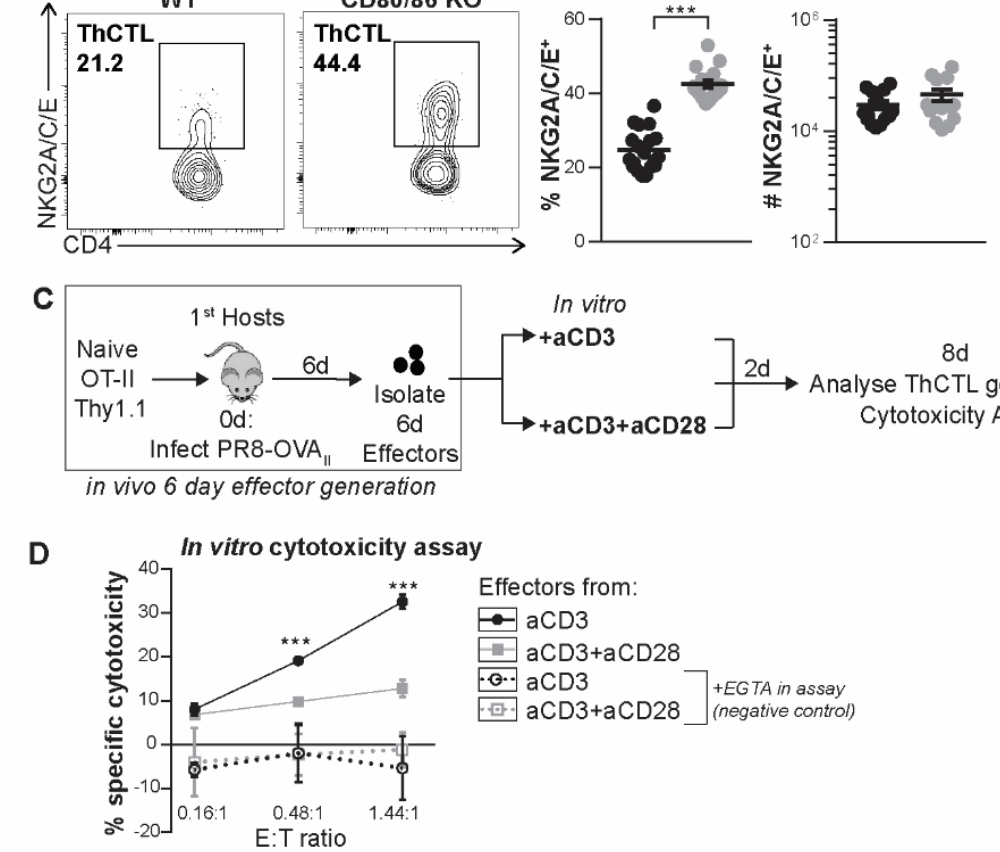

Effectors from:

$-\rightarrow \operatorname{aCD} 3$

aCD3+aCD28

$-9 \cdot \mathrm{aCD} 3$

+EGTA in assay

E

Gated on Spleen donor cells
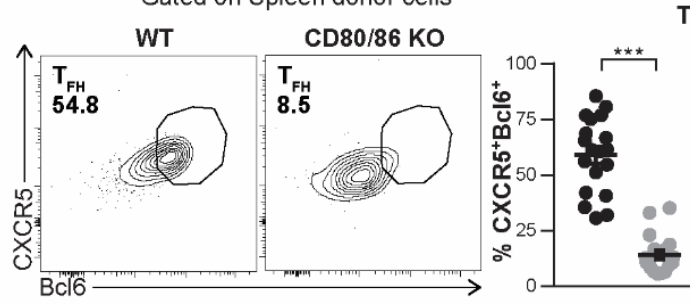

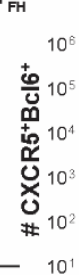

- CD80/86 KO •WT

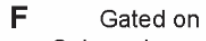

Spleen donor cells

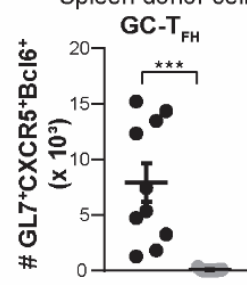

Fig. 3. $\mathrm{T}_{\mathrm{FH}}$ and ThCTL have different effector phase CD28 co-stimulation requirements. (A) Experimental design for (B,E-F): In vivo generated 6d OT-II.Thy $1.1^{+}$effectors were transferred into 6 dpi PR8-OVA -infected $_{\text {II }}$ WT or CD80/CD86-- mice. Spleen, DLN and lungs were harvested at 8 dpi. (B) Percentage and number of lung donor ThCTL (NKG2A/C/E $)$ ( $\mathrm{n}=14-19$ per group pooled, 4 independent experiments). (C) Experimental design: In vivo generated 6d OT-II.Thy $1.1^{+}$effectors were isolated and stimulated with either anti-CD3 alone or anti-CD3 and anti-CD28 in vitro to mimic in vivo effector phase cognate Ag stimulation. (D) Ag specific cytotoxicity of donors generated as in Fig. 3C, with anti-CD3 or anti-CD3 + anti-CD28 (Each E:T ratio is assayed in triplicate or single wells for +EGTA conditions, representative of 2 independent experiments). (E-F) Experiment done as in Fig. 3A. (E) Percentage and number of spleen donor $\mathrm{T}_{\mathrm{FH}}(\mathrm{n}=14-19$ per group pooled, 3-4 independent experiments). (F) Number of spleen donor $\mathrm{GC}-\mathrm{T}_{\mathrm{FH}}\left(\mathrm{GL} 7^{+} \mathrm{CXCR} 5^{+} \mathrm{Bcl} 6^{+}\right)(\mathrm{n}=8-10$ per group pooled, 2 independent experiments). Error bars represent s.e.m. Statistical significance determined by two-tailed, unpaired Student's t-test $(* \quad \mathbf{P}<0.05, \quad * * \quad \mathbf{P}<0.01 \quad$ and $* * * \quad \mathbf{P}<0.001)$. See also Fig. S4. 
ThCTL developed when effectors were stimulated by CD3 alone and adding CD28 co-stimulation inhibited ThCTL generation (Figure S4D). Cytotoxic function also depended on anti-CD3 stimulation and was inhibited when CD28 co-stimulation was provided in vitro (Figure 3D). Together the in vitro and in vivo results indicate that ThCTL differentiation from $6 \mathrm{~d}$ effectors does not require CD28 co-stimulation. The lack of a need for CD28 co-stimulation is also reminiscent of human ThCTL that studies have defined as CD28 negative populations (Serroukh et al., 2018; van de Berg et al., 2008).

In the same experiments (Figure $3 \mathrm{~A}$ ), both the proportion and absolute number of donor $\mathrm{T}_{\mathrm{FH}}$ and $\mathrm{GC}_{\mathrm{FH}}$ in the spleen and DLN were dramatically lower in the CD80/86 KO hosts (Figure 3E-F, Figure S4E-F). This agrees with previous data showing that CD28 co-stimulation post priming is necessary for $\mathrm{T}_{\mathrm{FH}}$ generation and maintenance (Linterman et al., 2014). Thus, CD28 co-stimulation of CD4 effectors at the effector checkpoint is required for full development of $\mathrm{T}_{\mathrm{FH}}$ in the spleen

272 and the DLN but is not required to sustain or induce further ThCTL generation in the lung. This

273 indicates that while both pathways of tissue-restricted effector development require Ag, the two

274 have distinct co-stimulation requirements during the effector phase. This is consistent with a 275 potential for multiple fate decisions taking place at this effector checkpoint, depending on the 276 details of the cognate interactions.

277 Multiple APC subsets can effectively present Ag at the effector checkpoint to drive $\mathbf{T}_{\mathrm{FH}}$ and

\section{ThCTL development}

279 We wondered if the distinct CD28 co-stimulation requirements for ThCTL and $\mathrm{T}_{\mathrm{FH}}$ subset 280 development might reflect a requirement for distinct APC subsets. To evaluate the efficacy of different broad classes of APC at the effector checkpoint, we used MHC-II KO bone marrow 
A

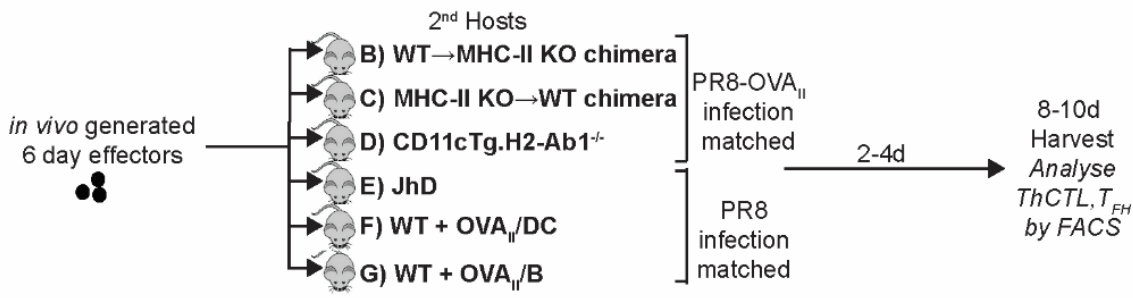

B $6 \mathrm{~d}$ effectors $\bullet \mathrm{WT} \rightarrow \mathrm{WT}$ chimera (Control) $\circ \mathrm{WT} \rightarrow \mathrm{MHC}-\mathrm{II}$ KO chimera (MHC-II only on hematopoietic cells)

Gated on donor cells

288

289

290

291

292

293

294

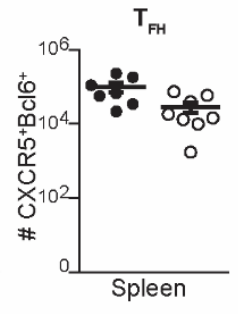

E 6d effectors - WWT

(B cells absent)
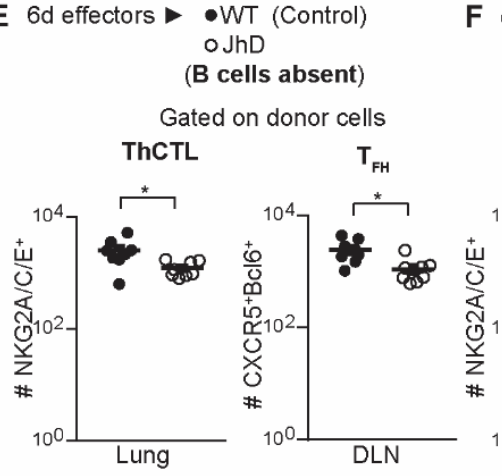
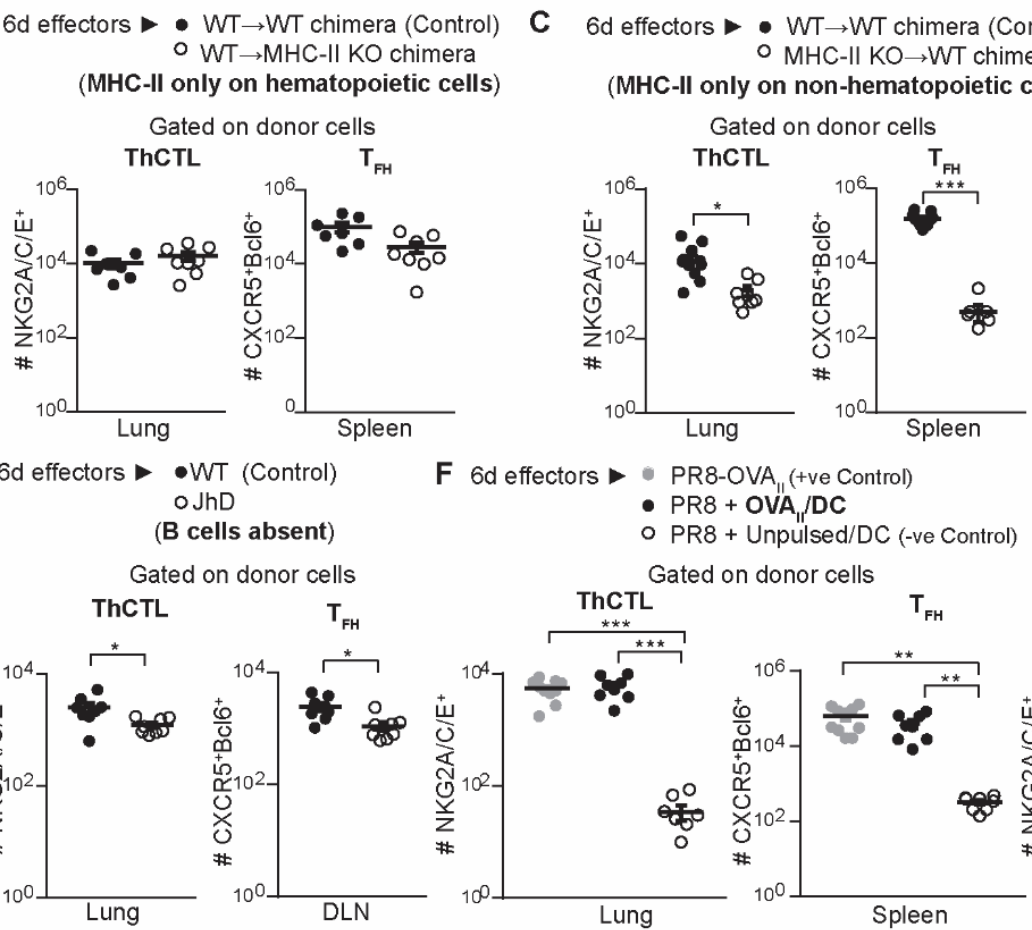

(MHC-II only on CD11c cells)

Gated on donor cells

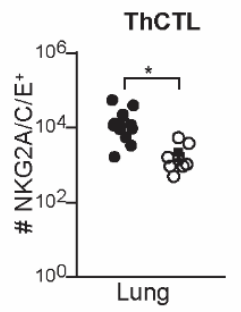

ThCTL
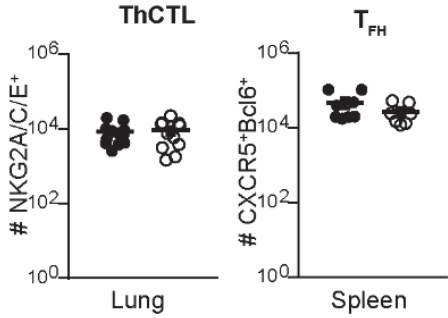

G 6d effectors $-\mathrm{PR}^{-O \mathrm{OA}_{\mu}}$ (+ve Control)

- $\mathrm{PR} 8+$ OVA $/ \mathrm{B}$

- $\mathrm{PR} 8+\mathrm{OVA}_{\mathrm{u}} / \mathrm{DC}$

- PR8 + Unpulsed/DC (-ve Control)

- PR8 + Unpulsed/B (-ve Control)

Gated on donor cells
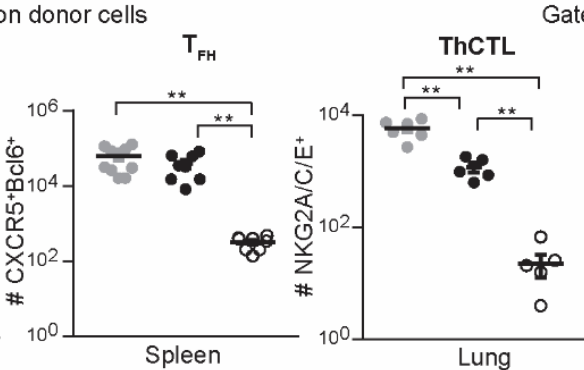

$\mathrm{T}_{\mathrm{FH}}$

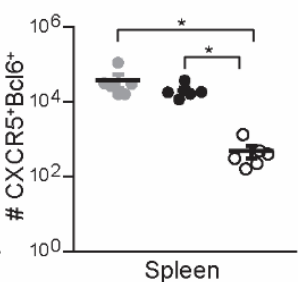

Fig. 4. Multiple APC subsets are able to present cognate Ag during the effector phase to support $\mathrm{T}_{\mathrm{FH}}$ and ThCTL generation from 6d effectors. (A) Experimental design: In vivo generated $6 \mathrm{~d}$ OT-II.Thy $1.1^{+}$or $6 \mathrm{~d}$ HNT. Thy $1.1^{+}$effectors were transferred into PR8-OVA ${ }_{\text {II }}$ infection-matched hosts (B-D), PR8 infection-matched hosts (E), or into PR8 infection-matched hosts together with $\mathrm{OVA}_{\mathrm{II}} / \mathrm{APC}(\mathrm{F}-\mathrm{G})$. Numbers of $\mathrm{T}_{\mathrm{FH}}\left(\mathrm{CXCR} 5^{+} \mathrm{Bcl} 6^{+}\right)$ and ThCTL (NKG2A/C/E $)$ generated were enumerated by flow cytometry, 2-4 dpt in each of these models. (B) $\mathrm{WT} \rightarrow \mathrm{MHC}-\mathrm{II} \mathrm{KO}\left(\mathrm{H} 2-\mathrm{Ab}^{--}\right)$bone marrow chimera mice that were made by transferring WT bone-marrow into MHC-II KO irradiated hosts, where MHC-II is restricted to the hematopoietic compartment, or into WT $\rightarrow$ WT bone marrow chimera control mice ( $\mathrm{n}=7-8$ per group pooled, 3 independent experiments). (C) MHC-II KO $\rightarrow$ B6 bone marrow chimera mice, where MHC-II is restricted to the non-hematopoietic compartment, or into $\mathrm{WT} \rightarrow \mathrm{WT}$ bone marrow chimera control mice ( $\mathrm{n}=8-11$ per group pooled, 3 independent experiments). (D) CD11cTg.H2-Ab $1^{-/}$mice where MHC-II is restricted to $\mathrm{CD} 11 \mathrm{c}^{+}$cells or into $\mathrm{CD} 4 \mathrm{KO}$ control mice $(\mathrm{n}=7-11$ per group pooled, 2-3 independent experiments). (E) JhD mice where B cells are absent or into WT control mice ( $\mathrm{n}=8$ per group pooled, 2 independent experiments). (F) WT mice with cognate Ag supplied via OVA $\mathrm{AII}_{\text {II }}$ pulsed BMDC vs unpulsed BMDC controls ( $\mathrm{n}=8-10$ per group pooled, 3 independent experiments). (G) WT mice with cognate Ag supplied via OVA II pulsed B cells vs unpulsed B cell controls ( $n=5-6$ per group pooled, 2 independent experiments). Error bars represent s.e.m. Statistical significance determined by two-tailed, unpaired Student's t-test $\quad{ }^{*} \quad \mathrm{P}<0.05, \quad * * \quad \mathrm{P}<0.01 \quad$ and $\left.\quad * * * \quad \mathrm{P}<0.001\right)$ See also Fig. S5. 
302 We transferred 6d effectors into infection-matched BM chimeras in which MHC II Ag-

303 presentation was restricted to either the hematopoietic compartment [WT $\rightarrow$ MHC-II KO

304 chimeras] (Figure 4B, Figure S5A) or to the non-hematopoietic compartment [MHC-II KO $\rightarrow$ WT

305 chimeras] (Figure 4C, Figure S5B). There was no defect in ThCTL generation when MHC-II was

306 restricted to the hematopoietic compartment. A substantial ThCTL population was also generated

307 when MHC-II was restricted to non-hematopoietic cells though we found significantly fewer donor

308 ThCTL (Figure 4C). Since a substantial ThCTL population was generated in both chimeras

309 (Figure 4B-C, Figure S5A-B), it suggests that both hematopoietic and non-hematopoietic APC can

310 present the Ag, to drive ThCTL development at the effector checkpoint. MHC-II is upregulated on

311 infected epithelial cells in the lung during IAV infection, so they may be a source of non-

312 hematopoietic APC (Brown et al., 2012). Donor $\mathrm{T}_{\mathrm{FH}}$ were found when Ag was restricted to the

313 hematopoietic compartment (Figure 4B, Figure S5A). In contrast, few if any $\mathrm{T}_{\mathrm{FH}}$ were found when

314 Ag presentation was restricted to the non-hematopoietic compartment (Figure 4C, Figure S5B),

315 consistent with few non-hematopoietic $\mathrm{MHC}-\mathrm{II}^{+}$cells presenting Ag in the SLO (Malhotra et al.,

316 2013).

317 Since the hematopoietic compartment was sufficient to support Ag presentation to both $\mathrm{T}_{\mathrm{FH}}$ and

318 ThCTL, we next asked if either of the classic APC: B cells and DCs, would be sufficient to drive

319 the tissue-restricted effectors. We restricted Ag-presentation to $\mathrm{CD}_{11 \mathrm{c}^{+}} \mathrm{APCs}$ by using

320 CD11cTg.H2-Ab1 $1^{-/}$mice (Figure 4D, Figure S5C). There was no defect in either donor ThCTL

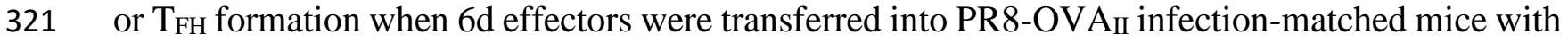

322 MHC-II only on CD11 $\mathrm{c}^{+}$APC, indicating that CD11c $\mathrm{c}^{+}$APC are sufficient to drive both ThCTL

323 and $\mathrm{T}_{\mathrm{FH}}$ during the effector checkpoint. 
$324 \mathrm{~B}$ cells are the major APC for $\mathrm{T}_{\mathrm{FH}}$ differentiation once they arrive in the follicular region of the

325 SLO (Krishnaswamy et al., 2018). To test whether B cells were necessary as APC for driving

326 tissue-restricted effectors during the effector checkpoint, we transferred in vivo generated 6d HNT

327 Thy1.1 (TCR Tg specific for HA epitope of the influenza strain) effectors into PR8 infection-

328 matched B cell deficient JhD mice (Figure 4E, Figure S5D). Substantial numbers of ThCTL and

$329 \mathrm{~T}_{\mathrm{FH}}$ were generated in the $\mathrm{B}$ cell deficient $\mathrm{JhD}$ hosts, though in both cases there was a two-fold

330 decrease in the number. This suggests that although B cell do contribute during the effector phase

331 as APC, non-B cells can also serve as APC for both ThCTL and $\mathrm{T}_{\mathrm{FH}}$ pathways.

332 We evaluated the impact of providing Ag on two different professional APC subsets: DC (Figure 4F, Figure S5E) and B cells (Figure 4G, Figure S5F). OVAII/APC were transferred together with

334 in vivo generated $6 \mathrm{~d}$ effectors into PR8 infection-matched mice. The $6 \mathrm{~d}$ effectors gave rise to 335 ThCTL and $\mathrm{T}_{\mathrm{FH}}$ when either B cells or DC presented Ag (Figure 4F-G, Figure S5E-F). We have 336 established previously that the OVA $/$ /DC present $\mathrm{Ag}$ for less than $2 \mathrm{~d}$ in vivo (Bautista et al., 2016).

337 The OVAII/DC transfer experiments suggest that, as for memory generation (Bautista et al., 2016), Ag recognition for the generation of $\mathrm{T}_{\mathrm{FH}}$ and $\mathrm{ThCTL}$, is only required for less than $2 \mathrm{~d}$ after effector transfer, indicating a short window for Ag recognition and a temporally-defined checkpoint.

341 including activated professional APC, such as DC and B cells, as well as non-hematopoietic cells 342 in infected mice. $\mathrm{T}_{\mathrm{FH}}$ were generated in all experiments where hematopoietic $\mathrm{MHC} \mathrm{II}^{+}$ 343 presentation was available to the $6 \mathrm{~d}$ effectors during the effector checkpoint. Thus, development 344 of both CD4 tissue effectors during the effector phase, is independent of any unique APC type, 345 with multiple APC able to be drive these fates. 
We hypothesized that Ag recognition during the effector checkpoint might be required in the site of residence to drive tissue-restricted effectors and that this might act to establish residency. To evaluate this for ThCTL generation, we first tested whether intranasal (i.n.) delivery of Ag/APC could target Ag presentation to the lung. We also transferred Ag/APC intrasplenically (i.s.) to exclude Ag presentation in the lung.

\begin{tabular}{|c|c|c|c|c|c|c|}
\hline & \multicolumn{4}{|c|}{} & \multicolumn{3}{c|}{$\begin{array}{c}\text { APC localization } \\
\text { Nur77 } \\
\text { Where are the OT-II that just saw Ag? }\end{array}$} \\
\cline { 2 - 8 } & i.n. & i.v. & i.s. & i.n. & i.v. & i.s. \\
\hline Spleen & $x$ & $\checkmark$ & $\checkmark$ & $x$ & $\checkmark$ & $\checkmark$ \\
\hline DLN & \multicolumn{3}{|c}{} & $\checkmark$ & $\checkmark$ & $x$ \\
\hline Lung & $\checkmark$ & $x$ & $x$ & $\checkmark$ & $\checkmark$ & $x$ \\
\hline
\end{tabular}

Table 1. Distribution of APC and Ag presentation in different sites using intranasal (i.n.), intravenous (i.v.) or intrasplenic (i.s.) transfer of APC. Data summarized from Fig 5B-C, 5F-G, 6A, 6E

We evaluated the localization of the APC using i.n. and i.s. delivery, and also evaluated corresponding Ag recognition by the transferred OT-II in the different sites (Table 1). To evaluate Ag recognition by effectors in the different sites using Nur77 $7^{\mathrm{GFP}}$ expression, we transferred OTII.Nur $77^{\mathrm{GFP}}$.Thy $1.1^{+}$6d effectors into PR8 infection-matched hosts with the OVAII/APC and analyzed them 14-16hr post transfer (Figure 5A). Only i.n. transfer of OVA Nur $77^{\mathrm{GFP}}$ expression in donor effectors in the lung while i.s. transfer did not induce Nur77 $7^{\mathrm{GFP}}$ expression over the negative control where APC without Ag (unpulsed APC) were transferred (Figure 5B). In concert with the site of $\mathrm{Ag}$ recognition as seen with Nur77 ${ }^{\mathrm{GFP}}$ expression, $\mathrm{OVA}_{\text {III }} / \mathrm{APC}$ were found only in the lung with i.n. transfer and only in the spleen with i.s. transfer (Figure 5C). These results indicate that we achieved localization of APC and successfully restricted $\mathrm{Ag}$ presentation to the lung with i.n. transfer of $\mathrm{Ag} / \mathrm{APC}$ and that i.s. transfer of $\mathrm{Ag} / \mathrm{APC}$ serves as a control where Ag is not presented locally in the lung (Table 1). 
368 We then analyzed ThCTL generation from the $6 \mathrm{~d}$ effectors, 3 days after transfer, at 9 dpi. Both i.n. and i.s. Ag/APC transfer increased trafficking of total transferred effectors to the lung, compared to the negative control (Figure 5D). We compared ThCTL generation after i.n. vs i.s. Ag/APC

371 delivery (Figure 5E). Strikingly, only i.n. delivery induced ThCTL. When we used i.s. delivery,

372 few if any ThCTL were generated, suggesting that Ag in the lung is required for lung ThCTL 373 generation.

374 To test if peripheral Ag was sufficient to drive 6d effectors to ThCTL, we transferred OVA II/APC 375 i.v. and compared ThCTL generation to i.n. OVA $\mathrm{II} / \mathrm{APC}$ transfer. OT-II.Nur77 ${ }^{\mathrm{GFP}}$. Thy $1.1^{+} 6 \mathrm{~d}$ 376 effectors were also transferred into PR8 infection matched hosts (Figure 5A). 14-16hr after 377 transfer, donor effectors in the lung expressed Nur77 ${ }^{\mathrm{GFP}}$ when OVA $\mathrm{II} / \mathrm{APC}$ were transferred either 378 i.v. or i.n., indicating Ag recognition (Figure 5F). A greater proportion of donor effectors in the 379 lung expressed Nur77 ${ }^{\text {GFP }}$ in hosts that received i.v. OVA $/$ IIPC compared to those that received 380 i.n. OVA 381 fewer in the lung with i.v. APC transfer (Figure 5G). This is compatible with the hypothesis that 382 after i.v. OVAII/APC transfer, donor effectors initially recognize $\mathrm{Ag}$ in the periphery before migrating to

the lung (Table $3841)$.

$385 \mathrm{OVA}_{\mathrm{II}} / \mathrm{APC}$ transfer i.v., like i.s., increased trafficking of transferred effectors to the lung, 386 compared to the negative control and even compared to i.n. OVA $\mathrm{II} / \mathrm{APC}$ transfer (Figure $5 \mathrm{H}$ ). 387 These data (Figure 5D, Figure 5H) support the concept, that Ag presentation in the spleen enhances pathways that favor migration of $\mathrm{T}$ cells to the lung. 


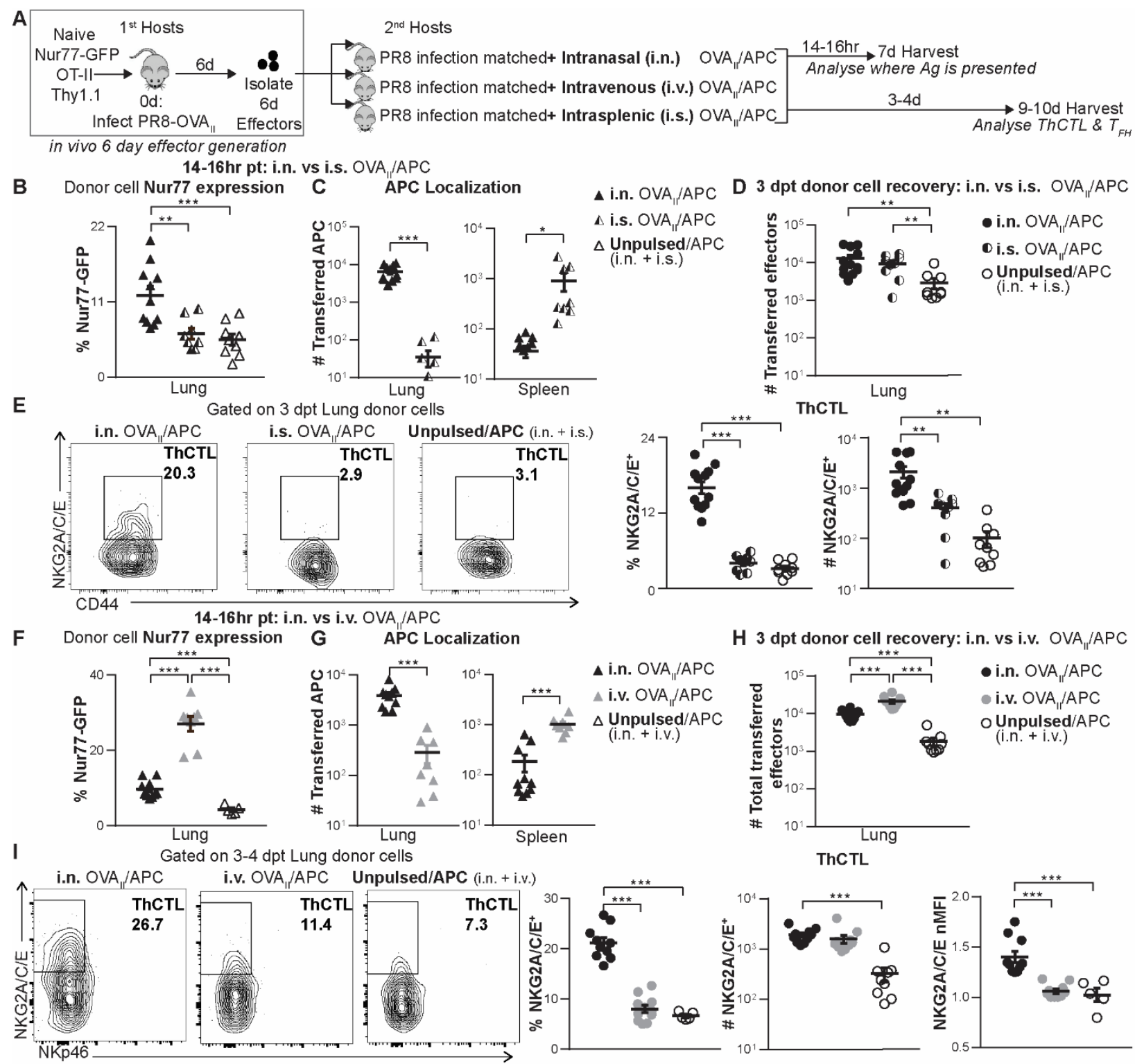

Fig. 5. Ag delivery via i.n., i.s and i.v. routes, during the effector phase, shows that local Ag presentation in the lung drives ThCTL generation from effectors. (A) Experimental design: OVA $\left(\mathrm{CD} 45.1^{+}\right.$or $\left.\mathrm{GFP}^{+}\right)$were used as APC and transferred into PR8 infection-matched hosts 6 dpi either intranasally (i.n.), intrasplenically (i.s.) or intravenously (i.v.). Unpulsed APC were transferred both i.n. and i.s. (B-E) or i.n. and i.v. (F-I) as negative controls. In vivo generated $6 \mathrm{~d}$ OT-II.Nur $77^{\text {GFP }}$. Thy $1.1^{+}$effectors were transferred i.v. Mice were harvested 14-16hr post-transfer (pt) and donor cells were analyzed by flow cytometry. (B) Donor Nur $77^{\mathrm{GFP}}$ expression (i.n. vs i.s. APC). (C) Number of transferred APC (i.n. vs i.s.) (D-E) Experiment performed as in (A) and mice harvested 3-4 dpt. (D) Number of donor effectors recovered with i.n. vs i.s. APC transfer. (E) Lung donor ThCTL formation with i.n. vs i.s. APC transfer. (F-H) Experiment performed as in (A). Mice harvested 14-16hr post-transfer (pt) (F) Donor Nur77 ${ }^{\mathrm{GFP}}$ expression (i.n. vs i.v. APC) (G) Number of transferred APCs (i.n. vs i.v.). (H-I) Experiment performed as in (A). Mice were harvested 3-4 dpt. (H) Number of lung donor effectors with i.n. vs i.v. APC.(I) Donor lung ThCTL formation with i.n. vs i.v. APC. (B-C, F-G: $n=8-11$ per group pooled, 3 independent experiments. D-E, H-I: $n=5-12$ per group pooled, 2-4 independent experiments) Error bars represent s.e.m. Statistical significance determined by two-tailed, unpaired Student's t-test $(* \mathrm{P}<0.05$, $* * \quad \mathrm{P}<0.01$ and $* * * \quad \mathrm{P}<0.001)$. See also Fig S6. 
Development of donor ThCTL in the lung as measured by NKG2A/C/E expression (both percent and MFI) after i.v. transfer, was as low as the negative control (Figure 5I). CXCR6 and PD1 expression by the $\mathrm{NKG} 2 \mathrm{~A} / \mathrm{C} / \mathrm{E}^{+}$cells generated with i.v. OVA $/ \mathrm{APC}$ transfer was also lower compared to those generated with i.n. OVA $\mathrm{II} / \mathrm{APC}$ (Figure S6A). This suggests that i.v. $\mathrm{OVA}_{\text {II }} / \mathrm{APC}$ did not optimally support full ThCTL differentiation even if $6 \mathrm{~d}$ effectors recognize when $\mathrm{Ag}$ is presented in the tissue of residency, the lung.

\section{Delivery of Ag by different routes favors Ag presentation in distinct SLO during the effector}

$\mathrm{T}_{\mathrm{FH}}$, like ThCTL, are restricted to the SLO (DLN and spleen) and express signatures for residency in SLO (Fazilleau et al., 2009; Lee et al., 2015). We asked if DLN and spleen $\mathrm{T}_{\mathrm{FH}}$ required Ag presentation in their organ of residence to drive their development during the effector phase. Using the same approach for evaluating local Ag requirements as for ThCTL in Figure 5, we delivered Ag via OVA $\mathrm{II} / \mathrm{APC}$ either i.n. or i.s. and transferred OT-II.Nur $77^{\mathrm{GFP}}$. Thy $1.1^{+} 6 \mathrm{~d}$ effectors into PR8 infection-matched hosts (Table 1). Transfer of OVA DLN and not in the spleen, while i.s. transfer induced Nur $77^{\mathrm{GFP}}$ exclusively in donor cells

407 recovered from the spleen and not in the DLN (Figure 6A). These results indicate that we had successfully restricted Ag presentation using i.n. vs i.s. delivery to the either the DLN or the spleen respectively (Table 1).

We analyzed $\mathrm{T}_{\mathrm{FH}}$ generation from the $6 \mathrm{~d}$ effectors, 3 days after transfer, at 9 dpi. The total number with i.s. OVA $A_{I I} / A P C$ transfer and vice-versa for the number of donor cells recovered in the spleen 
413 (Figure 6B). We found that $\mathrm{T}_{\mathrm{FH}}$ in the DLN developed from the $6 \mathrm{~d}$ effectors, only when

$414 \mathrm{OVA}_{\mathrm{II}} / \mathrm{APC}$ were administered i.n. and not i.s. (Figure 6C). Conversely, spleen $\mathrm{T}_{\mathrm{FH}}$ were supported

415 only when $\mathrm{OVA}_{\mathrm{II}} / \mathrm{APC}$ were delivered i.s. and not when delivered i.n. (Figure 6D). Thus in the

416 same experiment (Fig 5-6), $\mathrm{T}_{\mathrm{FH}}$ like ThCTL, develop only when Ag is presented in the tissue of

417 residencv

418

419

420

A $\quad 14-16 \mathrm{hr}$ pt: i.n. vs i.s. $O V A_{\|} / \mathrm{APC}$ Donor cell Nur77 expression

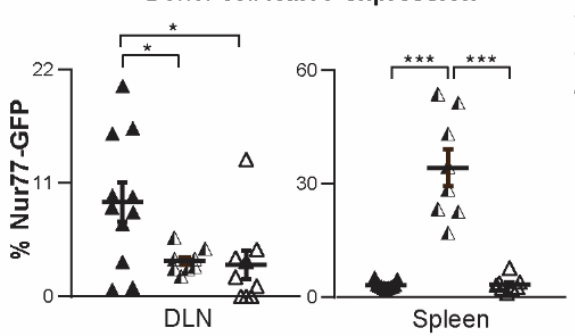

$\triangle$ i.n. $O V A_{\|} / A P C$

$\triangle$ i.s. $O V A_{\|} / A P C$

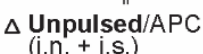

C

i.n. OVA /APC Gated on 3 dpt DLN donor cells

421

422

423

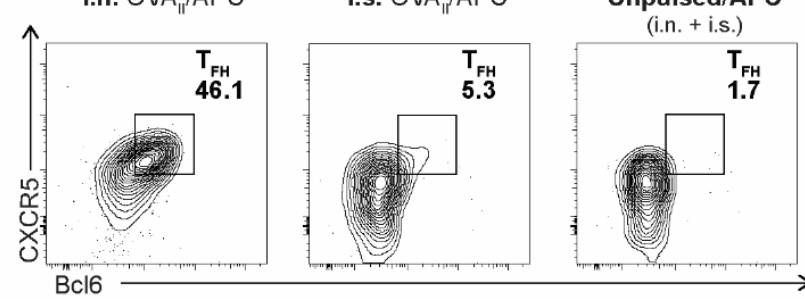

424

425

426

427

D Gated on 3 dpt Spleen donor cells

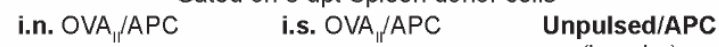

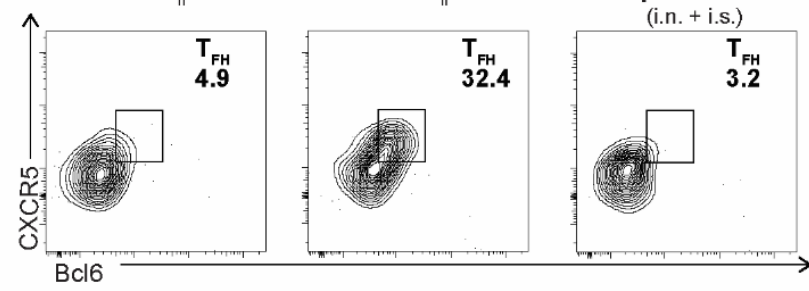

428

429

430

431

432

i.n. $O V A_{\|} / A P C$

$\triangle$ i.v. $O V A_{11} / A P C$

$\triangle$ Unpulsed/APC
E 14-16hr pt: i.n. vs i.v. OVA $_{1 /} / A P C$
Donor cell Nur77 expression

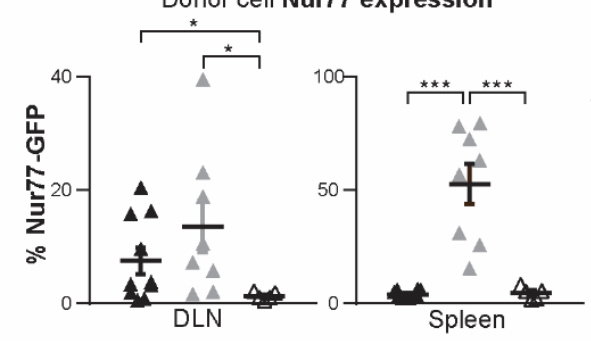

G
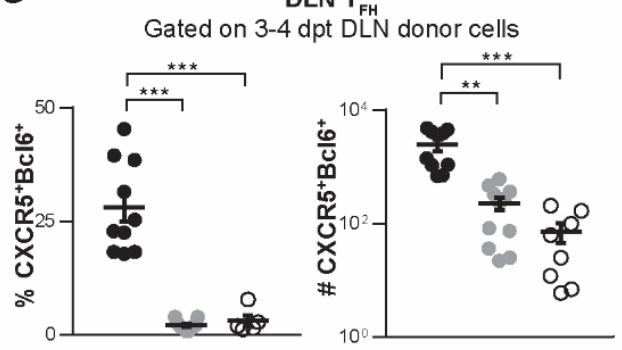

B $3 \mathrm{dpt}$ donor cell recovery

i.n. vs i.s. $O V A_{\|} / A P C$

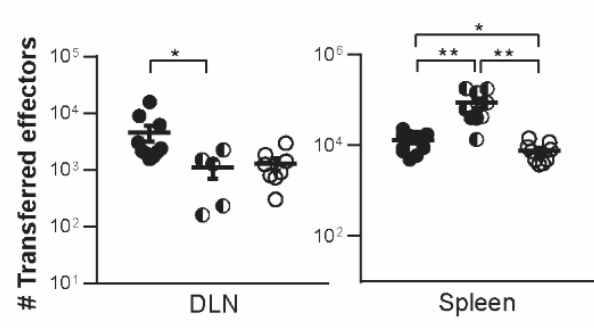

- i.n. $O V A_{\|} / A P C$

- i.s. $O V A_{\|} / A P C$

(i.n. + i.s.)

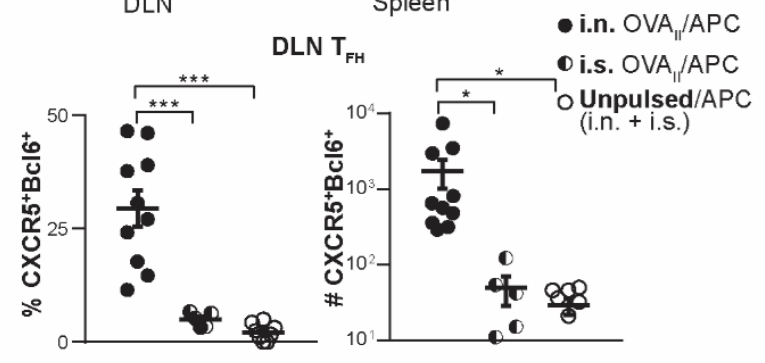

Spleen $\mathrm{T}_{\mathrm{FH}}$

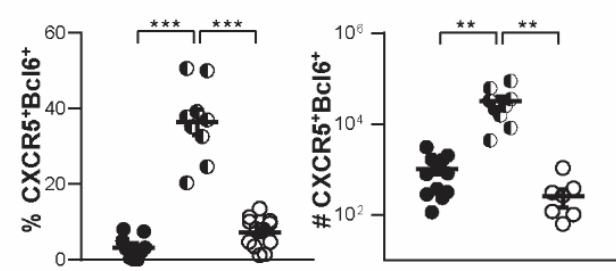

F $\quad 3 \mathrm{dpt}$ donor cell recovery

i.n. vs i.v. $O V A_{1 /} / A P C$

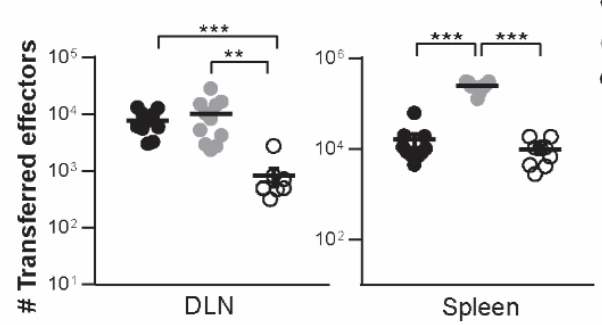

- i.n. $O V A_{\|} / A P C$

- i.v. OVA $/$ /APC

O Unpulsed/APC

Spleen $T_{F H}$

Gated on 3-4 dpt Spleen donor cells

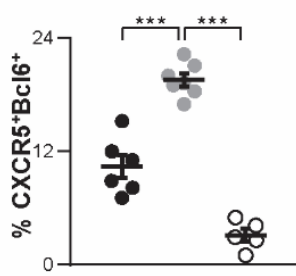

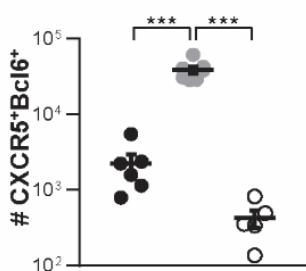

- i.n. $O V A_{\|} / A P C$

- i.v. OVA $/$ /APC

○ Unpulsed/APC

Fig. 6 
Fig. 6. DLN TFH and spleen $T_{F H}$ require local Ag presentation during the effector phase as shown by Ag delivery via i.n., i.s and i.v. routes (A) Experiment was performed as in Fig. 5A. Mice were harvested 14-16hr post-transfer (pt) and donor cell Nur77 ${ }^{\mathrm{GFP}}$ expression was analyzed by flow cytometry in the DLN and spleen (i.n. vs i.s. APC). (B-D) Experiment was performed as in Fig. 5A and mice were harvested 3-4 dpt. (B) Total numbers of DLN and spleen donor effectors recovered with i.n. vs i.s. APC transfer ( $n=5-12$ per group pooled, 3-4 independent experiments). (C) DLN donor $\mathrm{T}_{\mathrm{FH}}$ formation with i.n. vs i.s. APC transfer $(\mathrm{n}=5-10$ per group pooled, 3 independent experiments) (D) Spleen donor $\mathrm{T}_{\mathrm{FH}}$ formation with i.n. vs i.s. APC transfer $(\mathrm{n}=9-12$ per group pooled, 4 independent experiments). (E) Experiment was performed as in Fig. 5A. Mice were harvested 14-16hr post-transfer (pt) and donor cell Nur77 ${ }^{\text {GFP }}$ expression was analyzed by flow cytometry in the DLN and spleen (i.n. vs i.v. APC). (F-H) Experiment was performed as in Fig. 5A. Mice were harvested 3-4 dpt. (F) Numbers of donor effectors in the DLN and spleen when APC were transferred i.n. vs i.v. (n=5-11 per group pooled, 2-3 independent experiments). (G) Donor $\mathrm{T}_{\mathrm{FH}}$ formation in the DLN when APC were transferred i.n. vs i.v. $(\mathrm{n}=5-11$ per group pooled, 2-3 independent experiments). (H) Donor $\mathrm{T}_{\mathrm{FH}}$ formation in the spleen when APC were transferred i.n. vs i.v. ( $\mathrm{n}=5-11$ per group pooled, 2-3 independent experiments). Error bars represent s.e.m. Statistical significance determined by two-tailed, unpaired Student's t-test $(* \mathrm{P}<0.05, * * \mathrm{P}<0.01$

We also tested if peripheral Ag was sufficient to drive 6 dpi effectors to $\mathrm{T}_{\mathrm{FH}}$ in the SLO. To do this, we transferred OVAII/APC i.v. with OT-II.Nur77 ${ }^{\mathrm{GFP}}$. Thy $1.1^{+} 6 \mathrm{~d}$ effectors. After 14-16hr, donor effectors in both the spleen and DLN expressed Nur77 ${ }^{\mathrm{GFP}}$, indicating Ag recognition (Figure 6E, Table 1) though APC were found predominantly in the spleen with i.v. APC transfer (Figure 5G, Table 1). As seen for lung ThCTL, OVAII/APC transferred i.v. did not support donor DLN

$457 \mathrm{~T}_{\mathrm{FH}}$ generation but did support spleen $\mathrm{T}_{\mathrm{FH}}$ (Figure 6G-H, Figure S6B-C). This suggests that even 458 if 6d effectors recognize Ag initially before migrating to their site of residence in the DLN, this is 459 insufficient to induce development of DLN $\mathrm{T}_{\mathrm{FH}}$.

460 Altogether, these results support the concept that the final steps in full-fledged tissue-restricted ThCTL and $\mathrm{T}_{\mathrm{FH}}$ tissue-restricted effector generation from $6 \mathrm{~d}$ effectors, require local $\mathrm{Ag}$ recognition in the site of residency. 


\section{DISCUSSION}

465 While the instrumental role of Ag during the priming of T cells is well appreciated, we show here

466 that signals from local Ag and co-stimulation, during the effector phase, are required to drive

467 development of specialized $\mathrm{T}_{\mathrm{FH}}$ and ThCTL tissue-restricted effectors. These results indicate that

468 generation of these tissue-restricted effectors following influenza infection, unlike circulating Th1

469 effectors, require that infection continues into the effector phase to supply these Ag signals. Thus,

470 if infection is absent at this checkpoint, few of these specialized tissue effectors will develop.

471 Given the well-studied roles of ThCTL in viral clearance and $\mathrm{T}_{\mathrm{FH}}$ in subsequent $\mathrm{B}$ cell $\mathrm{Ab}$

472 responses (Crotty, 2019; Juno et al., 2017; Marshall and Swain, 2011; McKinstry et al., 2012), the

473 lack of these subsets will undermine a successful response. We postulate this checkpoint

474 mechanism acts as a safeguard to limit $\mathrm{T}_{\mathrm{FH}}$ and ThCTL generation to those situations where there

475 is an ongoing threat, thereby limiting unnecessary, potentially harmful responses. Indeed,

476 exaggerated $\mathrm{ThCTL}$ and $\mathrm{T}_{\mathrm{FH}}$ responses are seen in certain autoimmune diseases and chronic

477 infections (Broadley et al., 2017; Gensous et al., 2018) where continuous Ag and inflammation

478 persist.

479 Requirements for repeated/prolonged $\mathrm{Ag}$ for generation of $\mathrm{T}_{\mathrm{FH}}$ have been previously reported

480 (Krishnaswamy et al., 2018), but this has been unexplored for other tissue-restricted CD4 effector

481 subsets, such as ThCTL. It is known that $\mathrm{T}_{\mathrm{FH}}$ require repeated $\mathrm{Ag}$ recognition and costimulatory

482 interactions during priming and at the T-B border and in the GC of the SLO (Krishnaswamy et al.,

483 2018). Thus, it is expected though unknown if $\mathrm{T}_{\mathrm{FH}}$ will require $\mathrm{Ag}$ recognition, well into the late

484 effector phase as well. We show that this is indeed the case, but also show that the requirement of

485 CD4 effectors for $\mathrm{Ag}$ recognition at the checkpoint is not restricted to $\mathrm{T}_{\mathrm{FH}}$ but part of a broader

486 paradigm that drives multiple specialized CD4 responses, including $\mathrm{T}_{\mathrm{FH}}$, ThCTL and CD4 
487

488

489

490

491

492

493

494

495

496

497

498

499

500

501

502

503

504

505

506

507

508

509

memory. Importantly, we find that the cognate Ag requirement during the effector phase for both ThCTL and $\mathrm{T}_{\mathrm{FH}}$ can occur independently of both $\mathrm{B}$ cells and germinal centers. In addition, we show that CD4 effectors must recognize Ag in their tissue of residence to become tissue-restricted effectors.

ThCTL are not found until after 6 dpi (Marshall et al., 2016) and the $6 \mathrm{~d}$ effectors we transfer express no ThCTL markers (Figure S1E). Thus, we interpret their dependence on effector phase cognate-Ag recognition, as driving their generation from CD4 effectors. On the other hand, the $\mathrm{T}_{\mathrm{FH}}$ developmental program begins early during CD4 effector activation when effectors begin to express Bcl6 within the first few rounds of cell division (Vinuesa et al., 2016). Thus, the 6d effectors we transfer include some "pre- $\mathrm{T}_{\mathrm{FH}}$ " at $6 \mathrm{dpi}$ (Figure S1D). The $6 \mathrm{~d}$ pre- $\mathrm{T}_{\mathrm{FH}}$ have the potential to become $\mathrm{T}_{\mathrm{FH}}$, but our data here (25-fold reduction to negligible $\mathrm{T}_{\mathrm{FH}}$ levels in the absence of $\mathrm{Ag}$ ), suggest they only realize that potential if they receive signals from $\mathrm{Ag}$ recognition again, locally, during the effector phase. We plan further studies to explore which of the programs differentiation/generation, expansion, survival/maintenance - are induced by $\mathrm{Ag}$ recognition in this model. Altogether, what is evident from our functional data is that, in the absence of $\mathrm{Ag}$ presentation during the effector phase, there are few if any $\mathrm{T}_{\mathrm{FH}}$ that drive $\mathrm{GC}$ responses (Figure 2H-J) and few ThCTL that mediate MHC-II cytotoxicity (Figure 1E).

During polyclonal responses, new naïve CD4 T cells are recruited throughout the response (JelleyGibbs et al., 2005) and individual polyclonal cells have different propensities to become $\mathrm{T}_{\mathrm{FH}}$ because of their different TCRs (Krishnaswamy et al., 2018). These features create a nonsynchronized effector population which makes it difficult to identify cells at the same state of differentiation and track their fate. We circumvented these issues by generating effectors in situ from homogenous naïve TCR Tg CD4 T cells from two different models that gave corresponding 
results: B6.OT-II and BALB/c.HNT. Moreover, we have reproduced the strict requirement for Ag recognition by CD4 effectors for memory generation in a BALB/c D0.11.10 model, in this B6.OTII model (Bautista et al., 2016; McKinstry et al., 2014) and in a new influenza NP-specific TcR Tg. Usage of the TCR Tg sequential transfer model allowed the study of effector phase signals specifically, after 6 days post infection, which we could not have achieved using a polyclonal system.

Our experiments here focus solely on the requirements at the effector phase from 6 dpi onwards, coincident with the previously defined checkpoint for effectors in memory studies (Bautista et al., 2016; McKinstry et al., 2014). We identified several signals required to drive effectors at the checkpoint to $\mathrm{T}_{\mathrm{FH}}$ and ThCTL. First, effectors became $\mathrm{T}_{\mathrm{FH}}$ and ThCTL only when they recognized cognate Ag/APC at 6-8 dpi (Figure 1-2). Previously, we showed that CD4 memory generation required cognate Ag recognition 6-8 dpi and that this was dependent on autocrine IL-2 induction (Bautista et al., 2016; McKinstry et al., 2014). We confirmed the timeframe by blocking costimulatory pathways, blocking the IL-2 pathway and by adding back IL-2. The checkpoint coincides in situ with the peak of the effector response, which is followed by rapid contraction, supporting the concept that effectors express a default program of apoptosis which they avoid only when they recognize Ag (Bautista et al., 2016; McKinstry et al., 2014). We showed that the absence of effector checkpoint Ag results in poor CD4 memory development, corresponding with loss in protection against lethal influenza infection (Bautista et al., 2016). Thus, the development of three functionally specialized CD4 subsets, $\mathrm{T}_{\mathrm{FH}}$, ThCTL and CD4 memory, each requires cognate $\mathrm{Ag}$ recognition during this effector checkpoint. In addition to TCR triggering by $\mathrm{Ag}, \mathrm{T}_{\mathrm{FH}}$ but interestingly not ThCTL, required CD80/86 on the APC indicating distinct pathways are required for these distinct tissue-restricted effector subsets (Figure 3). 
533 Second, we showed various activated $\mathrm{MHC}-\mathrm{II}^{+} \mathrm{APC}$, including DC and B cells, drove donor effectors to develop into $\mathrm{T}_{\mathrm{FH}}$ and ThCTL (Figure 4) consistent with previous studies of APC subsets required during initial priming of $\mathrm{T}_{\mathrm{FH}}$ responses (Deenick et al., 2010; Deenick et al., 2011). In our experiments, in vivo transferred DC present Ag for less than 48hr (Bautista et al., 2016) indicating that effectors only need a brief period of TCR triggering. $\mathrm{T}_{\mathrm{FH}}$ were generated from 6d effectors even when Ag was presented only by DC (Figure 4F, Figure S5E) and even in the absence of $\mathrm{B}$ cells and GC in JhD $2^{\text {nd }}$ hosts (Figure 4E, Figure S5D). Thus, although B cells may be the major source of $\mathrm{Ag}$ for $\mathrm{T}_{\mathrm{FH}}$ in situ, other $\mathrm{MHC}-\mathrm{II}^{+} \mathrm{APC}$ are competent to drive $\mathrm{T}_{\mathrm{FH}}$ development at the effector phase. Given the critical importance of $\mathrm{T}_{\mathrm{FH}}$ to effective $\mathrm{B}$ cell immunity, this may allow development of strong $\mathrm{T}_{\mathrm{FH}}$ responses even if germinal center responses

543 are impaired or Ag-specific B cells are limited. For instance, this may be a useful strategy for the 544 immune system to be able to drive GC-independent B cell responses which benefit from $\mathrm{T}_{\mathrm{FH}}$ help, 545 such as reactivation of previously generated memory B cells (Inoue et al., 2018).

547 APC was delivered to the site of future residence (Figure 5-6). Recently, Ag presentation in tissues 548 has been implicated in development of both T and B resident memory subsets (Allie et al., 2019;

549 Khan et al., 2016; McMaster et al., 2018; Takamura et al., 2016). Our data suggest that local Ag presentation in the tissue site, establishes residency during the effector checkpoint. Previous 551 studies have largely focused on tissue-resident $\mathrm{T}$ cell subsets in non-lymphoid tissues, however 552 SLO also have distinct architecture and function (Lewis et al., 2019; Malhotra et al., 2013). Here 553 we show that SLO-resident $\mathrm{T}_{\mathrm{FH}}$ subsets also have unique local Ag presentation requirements 554 during the effector checkpoint (Figure 6) that are distinct from lung resident ThCTL and suggests 555 that tissue-restricted $\mathrm{Ag}$ presentation is required even if they encountered $\mathrm{Ag}$ before entering the 
local tissue niche (Figure 6E-H). A subset of SLO T $\mathrm{RM}_{\mathrm{R}}$ has also been identified recently (Beura et al., 2018; Schenkel et al., 2014). Thus, our results here and those recent studies, solidify the previously underappreciated concept that SLO also harbor tissue resident $\mathrm{T}$ cell subsets with unique requirements for differentiation.

The functional activity of $\mathrm{T}_{\mathrm{FH}}$ and ThCTL correlated well with the availability of the checkpoint signals. $\mathrm{T}_{\mathrm{FH}}$ required Ag recognition to produce IL-21 (Figure 2E) and to induce GCB (Figure 2H-

(Figure 1E). Tissue-restricted effector functions are critical to immunity (Devarajan et al., 2018).

571 viruses and tumor microenvironments where CD8 cytotoxic cells are ineffective. Tissue-restricted 572 effectors are also likely the precursors of $\mathrm{T}_{\mathrm{RM}}$ which are at the frontline of immune defense to re573 infection.

574 These results are directly relevant to vaccine design. The most common Ab viral epitopes on the 575 surface proteins shift frequently, constraining the ability of long-lived Ab produced by B cells, to 576 remain fully protective. Thus, T cells are central to broad immunity to influenza and other RNA 577 viruses that mutate because the they target core proteins of viruses that rarely change (Devarajan 578 et al., 2016). Our results suggest that vaccine approaches need to deliver Ag to tissues, such as 579 Flumist which delivers Ag to the lung, if they are to efficiently drive the tissue-restricted CD4 T 
580 cell subsets and CD4 memory. Additionally, unformulated soluble Ag/adjuvants in vaccines have

581 been shown to be rapidly cleared from the body (Moyer et al., 2016), which may explain the low

582 efficacy of current influenza vaccines in generating durable CD4 T cell responses and indirectly

583 strong long-term B cell immunity. Several formulation strategies have been proposed such as

584 synthetic polymer formulations, microneedle skin patches and polymer sponges to extend the

585 kinetics of Ag presentation in vaccines (Moyer et al., 2016). One recent strategy engineered 586 enhanced Ag binding on alum, which allowed Ag presentation well into the effector phase (Moyer

587 et al., 2020) and elicited superior humoral immunity. We predict that such strategies are likely to

588 better support tissue effector formation.

589 Coupled with our earlier studies of memory generation (Bautista et al., 2016; McKinstry et al., 590 2014), our results here support a new paradigm in which a set of critical fate decisions occur at the 591 CD4 effector checkpoint to coordinately support generation of multiple alternate fates: CD4 592 memory, $\mathrm{T}_{\mathrm{FH}}$ and ThCTL. We believe that the regulation of the response at the effector checkpoint 593 by cognate $\mathrm{Ag}$, can lend new perspective on mechanisms of autoimmune pathogenesis driven by 594 CD4 tissue effectors. We also suggest that to induce durable immunity, the most effective vaccines 595 must provide the effector checkpoint signals identified here at the right time and in the relevant 596 sites, so as to drive robust tissue-restricted effector as well as memory cell generation resulting in 597 both more effective immunity and more "universal" influenza protection (Devarajan et al., 2016). 


\section{ACKOWLEDGEMENTS}

603

604

605

606

607

608

609

610

611

612

613

614

615

616

617

618

619

620

621

622

623
We would like to thank Dr. Richard Dutton, Dr. Kai McKinstry, Dr. Tara Strutt and Dr. Esteban

Rozen for useful discussions and advice throughout the project. We would also like to thank Yi

Kuang, Jialing Liang and Mike Perkins for assistance with experiments and animal husbandry.

This work was supported by funding from grants U19 AI109858, P01 AI046530, R01 AI118820

and R21 AI128606 to S.L.S; T32 AI007349 to A.M.V., M.C.J. and O.K.U.; R25 GM113686 and T32 AI132152 to O.K.U.

\section{AUTHOR CONTRIBUTIONS}

P.D. and S.L.S. wrote the manuscript with assistance from A.M.V., B.L.B., M.C.J. and O.K.U. P.D., A.M.V. and S.L.S. conceived the project and designed experiments. P.D. and A.M.V. performed and analyzed experiments with assistance from C.H.C., B.L.B., M.C.J. and O.K.U. A.M.V. primarily performed and analyzed experiments for Figures 1-3 and associated supplementary figures. P.D. primarily performed and analyzed experiments for Figure 2, Figures 4-6 and associated supplementary figures. K.A.K. performed intrasplenic transfers with assistance from P.D. All authors have read and approved the submitted version.

\section{DECLARATION OF INTERESTS}

The authors declare no financial conflicts of interest. 

PR8-OVA II infected mice ( $1^{\text {st }}$ hosts). At 6 dpi, OT-II.Thy $1.1^{+}$effectors were isolated from $1^{\text {st }}$ hosts or uninfected mice. Donor cells were analyzed 8 dpi. (B) Percentage and numbers of donor lung histogram of lung donor cell GzmB expression (negative control: naïve CD4 from uninfected mice). Normalized MFI of lung donor cell GzmB expression ( $\mathrm{n}=10$ per group pooled, 2 independent experiments). (D) CD107a degranulation marker expression by lung donor cells ( $\mathrm{n}=9$ per group pooled, 2 independent experiments). (E) Experimental design: In vivo 6d OT-II.Thy $1.1^{+}$ effectors were transferred into 6 dpi PR8-OVA ${ }_{I I}$ or PR8 infection-matched TCR $\alpha / \beta^{-/-}$mice. CFSE ${ }^{\text {lo }}$ target and bystander $\mathrm{CFSE}^{\text {hi }}$ bystander cells were transferred at 7d. Representative CFSE histograms shown. Percentage Ag specific cytotoxicity in each group is shown. (F-G) Experiment done as in (E). Percentage of lung donor cells expressing intracellular IFN $\gamma(F)$ and TNFa (G) (EG, $n=7$ per group pooled, 2 independent experiments). Statistical significance determined by twotailed, unpaired Student's t-test $(* \mathrm{P}<0.05$, $* * \mathrm{P}<0.01, * * * \mathrm{P}<0.001)$. See also Figure $\mathrm{S} 2$.

Figure 2. SLO $\mathbf{T}_{\mathrm{FH}}$ require Ag recognition during the effector checkpoint. Experiment 642 performed as in Figure 1A for Figure 2A-G. (A) Percentage and numbers of spleen donor $\mathrm{T}_{\mathrm{FH}}$ $643\left(\mathrm{CXCR}^{+} \mathrm{Bcl6}^{+}\right)$. (B) Number of spleen donor germinal center $\mathrm{T}_{\mathrm{FH}}\left(\mathrm{GL}^{+} \mathrm{CXCR}^{+} \mathrm{Bcl6}^{+}\right)$. (C-D) 644 Representative histogram of ICOS (C) and PD1 (D) expression by spleen donor cells (negative control: naïve CD4 from uninfected mice). Normalized ICOS MFI (C) and PD1 MFI (D) 646 expression by spleen donor cells. (A-D, $n=10$ per group pooled, 2 independent experiments). (E- 

generated 6d OT-II.Thy $1.1^{+}$effectors were transferred into 2 dpi PR8-OVAII-infected or PR8infected mice. A group of 2 dpi PR8-OVAII-infected and PR8-infected mice, with no cells of host GCB cells $\left(\mathrm{CD} 19^{+} \mathrm{Fas}^{+} \mathrm{GL} 7^{+} \mathrm{Bcl}^{+}\right)$formed. (J) Percentage and numbers of $\mathrm{HA}^{+} \mathrm{GCB}$. (H$\mathrm{P}<0.001)$. See also Figure S3. were harvested at 8 dpi. (B) Percentage and number of lung donor ThCTL (NKG2A/C/E $)(n=14-$ 19 per group pooled, 4 independent experiments). (C) Experimental design: In vivo generated 6d OT-II.Thy $1.1^{+}$effectors were isolated and stimulated with either anti-CD3 alone or anti-CD3 and anti-CD28 in vitro to mimic in vivo effector phase cognate Ag stimulation. (D) Ag specific cytotoxicity of donors generated as in Figure 3C, with anti-CD3 or anti-CD3 + anti-CD28 (Each E:T ratio is assayed in triplicate or single wells for +EGTA conditions, representative of 2 independent experiments). (E-F) Experiment done as in Figure 3A. (E) Percentage and number of spleen donor $\mathrm{T}_{\mathrm{FH}}(\mathrm{n}=14-19$ per group pooled, 3-4 independent experiments). (F) Number of spleen donor $\mathrm{GC}-\mathrm{T}_{\mathrm{FH}}\left(\mathrm{GL}^{+} \mathrm{CXCR}^{+} \mathrm{Bcl6}^{+}\right)(\mathrm{n}=8-10$ per group pooled, 2 independent experiments). test $(* \mathrm{P}<0.05, * * \mathrm{P}<0.01$ and $* * * \mathrm{P}<0.001)$. See also Figure $\mathrm{S} 4$. 
674 hosts together with OVA $\mathrm{II} / \mathrm{APC}$ (F-G). Numbers of $\mathrm{T}_{\mathrm{FH}}\left(\mathrm{CXCR}^{+} \mathrm{Bcl6}^{+}\right)$and ThCTL

$675\left(\mathrm{NKG} 2 \mathrm{~A} / \mathrm{C} / \mathrm{E}^{+}\right)$generated were enumerated by flow cytometry, 2-4 dpt in each of these models.

(B) $\mathrm{WT} \rightarrow \mathrm{MHC}-\mathrm{II} \mathrm{KO}\left(\mathrm{H} 2-\mathrm{Ab}^{-/-}\right)$bone marrow chimera mice that were made by transferring

WT bone-marrow into MHC-II KO irradiated hosts, where MHC-II is restricted to the hematopoietic compartment, or into WT $\rightarrow$ WT bone marrow chimera control mice $(\mathrm{n}=7-8$ per group pooled, 3 independent experiments). (C) MHC-II KO $\rightarrow$ B6 bone marrow chimera mice, where MHC-II is restricted to the non-hematopoietic compartment, or into WT $\rightarrow \mathrm{WT}$ bone marrow $\mathrm{Ab}^{-/-}$mice where MHC-II is restricted to CD11c ${ }^{+}$cells or into CD4 KO control mice ( $\mathrm{n}=7-11$ per group pooled, 2-3 independent experiments). (E) JhD mice where B cells are absent or into WT control mice ( $\mathrm{n}=8$ per group pooled, 2 independent experiments). (F) WT mice with cognate Ag supplied via OVAII pulsed BMDC vs unpulsed BMDC controls ( $\mathrm{n}=8-10$ per group pooled, 3 unpulsed B cell controls ( $n=5-6$ per group pooled 2 independent experiments). Error bars represent s.e.m. Statistical significance determined by two-tailed, unpaired Student's t-test $(* \mathrm{P}<0.05$, ** $\mathrm{P}<0.01$ and $* * * \mathrm{P}<0.001)$. See also Figure S5.

Figure 5. Ag delivery via i.n., i.s and i.v. routes, during the effector phase, shows that local 
infection-matched hosts 6 dpi either intranasally (i.n.), intrasplenically (i.s.) or intravenously (i.v.). Unpulsed APC were transferred both i.n. and i.s. (B-E) or i.n. and i.v. (F-I) as negative controls. In vivo generated 6d OT-II.Nur77 ${ }^{\mathrm{GFP}}$.Thy $1.1^{+}$effectors were transferred i.v. Mice were harvested 14-16hr post-transfer (pt) and donor cells were analyzed by flow cytometry. (B) Donor Nur77 GFP expression (i.n. vs i.s. APC). (C) Number of transferred APC (i.n. vs i.s.) (D-E) Experiment performed as in (A) and mice harvested 3-4 dpt. (D) Number of donor effectors recovered with i.n. vs i.s. APC transfer. (E) Lung donor ThCTL formation with i.n. vs i.s. APC transfer. (F-G) Experiment performed as in (A). Mice harvested 14-16hr post-transfer (pt) (F) Donor Nur77 $7^{\mathrm{GFP}}$ expression (i.n. vs i.v. APC) (G) Number of transferred APCs (i.n. vs i.v.). (H-I) Experiment performed as in (A). Mice were harvested 3-4 dpt. (H) Number of lung donor effectors with i.n. vs i.v. APC. (I) Donor lung ThCTL formation with i.n. vs i.v. APC. (B-C, F-G: n=8-11 per group pooled, 3 independent experiments. D-E, H-I: n=5-12 per group pooled, 2-4 independent experiments) Error bars represent s.e.m. Statistical significance determined by two-tailed, unpaired Student's t-test $(* \mathrm{P}<0.05, * * \mathrm{P}<0.01$ and $* * * \mathrm{P}<0.001)$. See also Fig S6.

Figure 6. DLN TFH and spleen $T_{F H}$ require local Ag presentation during the effector phase as shown by Ag delivery via i.n., i.s and i.v. routes (A) Experiment was performed as in Figure 5A. Mice were harvested 14-16hr post-transfer (pt) and donor cell Nur77 ${ }^{\mathrm{GFP}}$ expression was analyzed by flow cytometry in the DLN and spleen (i.n. vs i.s. APC). (B-D) Experiment was performed as in Figure 5A and mice were harvested 3-4 dpt. (B) Total numbers of DLN and spleen donor effectors recovered with i.n. vs i.s. APC transfer ( $n=5-12$ per group pooled, 3-4 independent experiments). (C) DLN donor $\mathrm{T}_{\mathrm{FH}}$ formation with i.n. vs i.s. APC transfer ( $\mathrm{n}=5-10$ per group pooled, 3 independent experiments) (D) Spleen donor $\mathrm{T}_{\mathrm{FH}}$ formation with i.n. vs i.s. APC transfer ( $\mathrm{n}=9-12$ per group pooled, 4 independent experiments). (E) Experiment was performed as in 
716 Figure 5A. Mice were harvested 14-16hr post-transfer (pt) and donor cell Nur77 ${ }^{\text {GFP }}$ expression

717 was analyzed by flow cytometry in the DLN and spleen (i.n. vs i.v. APC). (F-H) Experiment was

718 performed as in Figure 5A. Mice were harvested 3-4 dpt. (F) Numbers of donor effectors in the

719 DLN and spleen when APC were transferred i.n. vs i.v. (n=5-11 per group pooled, 2-3 independent

720 experiments). (G) Donor $\mathrm{T}_{\mathrm{FH}}$ formation in the DLN when APC were transferred i.n. vs i.v. (n=5-

72111 per group pooled, 2-3 independent experiments). (H) Donor $\mathrm{T}_{\mathrm{FH}}$ formation in the spleen when

722 APC were transferred i.n. vs i.v. (n=5-11 per group pooled, 2-3 independent experiments). Error

723 bars represent s.e.m. Statistical significance determined by two-tailed, unpaired Student's t-test (*

$724 \mathrm{P}<0.05, * * \mathrm{P}<0.01$ and $* * * \mathrm{P}<0.001)$. See also Figure S6.

725

726

727

728

729

730

731

732

733

734

735 
Mice

C57B1/6 (B6), B6.CD45.1, B6.Thy1.1, B6.Nr4a1 ${ }^{\mathrm{eGFP}}\left(\mathrm{Nur77}{ }^{\mathrm{GFP}}\right)$, B6.CD80/CD86 KO and

B6.MHC II- were obtained from the Jackson Laboratory. B6.TCR $\alpha / \beta \mathrm{KO}$ mice were obtained from

Scripps Research Institute, La Jolla, CA) and were originally published by Frank Carbone’s group

743 (Barnden et al., 1998) and were bred and maintained at the UMMS animal facility. BALB/c.HNT weeks old prior to use.

Virus stocks and infections and A/PR8-OVA

750 Trudeau Institute. Mice were anesthetized with isoflurane (Piramal Healthcare) or with

751 Ketamine/Xylazine (at a dose of $25 / 2.5 \mathrm{mg} / \mathrm{kg}$ by i.p. injection) and were infected intranasally with

752 influenza virus corresponding to a $0.2-0.3 \mathrm{LD}_{50}$ dose of IAV in $50 \mathrm{uL}$ of PBS.

\section{Bone marrow chimera mice generation}

754 Host mice for bone marrow chimeras were lethally irradiated with 2 doses of 570 rads, 3 hours

755 apart. Bone marrow was isolated from the femurs and tibia of donor mice. The bone marrow was

756 T cell depleted (using CD90.2 magnetic beads from Miltenyi) and adoptively transferred into

757 lethally irradiated host mice by tail-vein i.v. injections. Bone marrow was transferred at a 1:1 or

758 1:2 donor:host mice ratio. Mice were allowed to recover and reconstitute for at least 6 weeks prior 
to use during which they were treated with antibiotics $(0.63 \mathrm{mg} / \mathrm{ml}$ Sulfadiazine and $0.13 \mathrm{mg} / \mathrm{mL}$ peripheral blood before use and again in all tissues harvested when the mice were used in experiments.

\section{In vivo day 6 effector generation and transfer/in vitro culture}

In vivo generated 6d CD4 T cell effectors were routinely obtained as described previously (Bautista et al., 2016). Briefly, cells from lymph nodes and spleens of naïve OT-II or HNT transgenic mice were enriched for naïve cells by percoll gradients and CD4 T cells isolated by CD4 positive selection (Miltenyi Biotec) or using a CD4 naïve positive selection kit (Miltenyi Biotec). Naïve

771 congenic marker (CD90.1). Immediately after isolation, the in vivo generated $1-2 \times 10^{6} 6 \mathrm{~d}$ CD4

772 effectors were adoptively transferred intravenously (i.v.) into host mice ( ${ }^{\text {nd }}$ hosts).

In vivo generated 6d CD4 effectors were also cultured in vitro for 2 days by stimulating with plate

774 bound anti-CD3 $(2 \mathrm{C} 11,0.5 \mathrm{ug} / \mathrm{ml})$ or anti-CD3 and anti-CD28 $(37.51,20 \mathrm{ug} / \mathrm{mL})$ in T cell media 775 (RPMI 1640 supplemented with $7.5 \%$ fetal bovine serum, 2mM L-glutamine, 50 uM 2776 mercaptoethanol, $100 \mathrm{IU}$ penicillin, $100 \mathrm{ug} / \mathrm{ml}$ streptomycin and 10mM HEPES).

779 and activated B cell (Bautista et al., 2016) generation was done as described previously. Briefly, bone marrow cells were flushed from femurs and tibia of mice and cultured in vitro with $10 \mathrm{ng} / \mathrm{mL}$ 
781 GMCSF (Biolegend). After 7 days, cells were harvested and enriched for dendritic cells with

782 CD11c positive selection (Miltenyi Biotec). Dendritic cells were then matured with 10ug/mL Poly I:C (InVivoGen) overnight before use. Activated B cells were generated by isolating T depleted splenocytes using CD90.2 negative selection (Miltenyi Biotec) and culturing these in vitro for 2 days with 10ng/mL LPS and 10ng/mL dextran sulfate.

\section{In vivo APC delivery}

787 To deliver Ag/APC (BMDC or activated B cells), APC were pulsed with 10 $\mu$ M OVA $323-339$ $\left(\right.$ OVA $_{\text {II }}$ peptide (New England Peptide) or no peptide as a negative control (unpulsed APC) for 1 hour at $37^{\circ} \mathrm{C}$ with shaking. APC were washed and administered either intravenously (i.v.) in $200 \mathrm{uL}$ PBS, intranasally (i.n.) in 50uL PBS, or intrasplenically (i.s.) in 10uL PBS. $0.25-1 \times 10^{6}$ BMDC or

For intrasplenic transfer of APC, the animal was initially anaesthetized at $2.5 \%$, then maintained at $1.5-1.75 \%$ isoflurane. Animal's hair was clipped from the hip to mid chest on the animal's left side. The area was sterilized and bupivacaine $1 \mathrm{mg} / \mathrm{kg}$ was subcutaneously injected at the proposed incision site. Just below the last rib, using a pair of forceps, a $2 \mathrm{~mm}$ area of skin was held up and away from the body cavity and a 6-8mm incision was made by blunt dissection. PBS soaked cotton tipped applicators were used to lift the spleen out and hold in place. A $25 \mu 1$ Hamilton syringe with a 31-gauge Hamilton needle was used to inject the cells into the spleen. Sterile PBS was drawn into the syringe 3 times prior to the cells being drawn up. The syringe was held in a vertical position

801 to the center of the spleen. The center of the top of spleen was penetrated by the needle at a depth 
804 placed back into the abdominal cavity. Muscle and skin layers were sutured closed. Upon 805 completion of the surgery, meloxicam SR $4.0 \mathrm{mg} / \mathrm{kg}$ was administered subcutaneously over the 806 right flank.

\section{T cell functional assays}

808

In vivo and in vitro cytotoxicity was performed as previously described (Marshall et al., 2016). Briefly, for in vivo cytotoxicity, T depleted splenocytes were stained with either 1uM or 0.4uM of CFSE denoting target $(0.4 \mathrm{uM})$ or bystander $(1 \mathrm{uM})$ cells. Target cells were pulsed with OVA $\mathrm{II}_{\mathrm{I}}$ peptide for 1 hour at $37^{\circ} \mathrm{C}$. Both populations were washed twice in PBS and adoptively transferred into host mice. 18 hours later, the spleens of host mice were harvested and the number of target and bystander cells were quantified by flow cytometry. Specific killing was calculated as: $100 \mathrm{x}$ (1- (live targets/live bystanders)) normalized to the ratio found in control mice. For in vitro cytotoxicity, targets were activated B cells that were labeled as above using CellTrace Violet (Invitrogen). Effectors and targets were co-cultured in $96 \mathrm{U}$ bottom plates in $\mathrm{T}$ cell medium at $37^{\circ} \mathrm{C} 5 \% \mathrm{CO}_{2}$ for 4 hours. Plates were washed and stained for cell viability using Annexin $\mathrm{V}$ and 7-AAD (Invitrogen) or live/dead amine dyes (Invitrogen). Ag specific cytotoxicity was calculated as: $100 \times$ (1- (live targets/live bystanders)) normalized to the ratio found in control wells with no effector cells. T cell degranulation and cytokine production was measured by ex vivo stimulation with plate bound $0.5 \mathrm{ug} / \mathrm{mL}$ anti-CD3 and $20 \mathrm{ug} / \mathrm{mL}$ anti-CD28 or with $10 \mathrm{ng} / \mathrm{mL}$ PMA and $500 \mathrm{ng} / \mathrm{mL}$ Ionomycin for 4 hours at $37^{\circ} \mathrm{C}, 5 \% \mathrm{CO}_{2}$ with brefeldin $\mathrm{A}(10 \mathrm{ug} / \mathrm{ml})$. $\mathrm{T}$ cell degranulation was also measured simultaneously with the addition of anti-CD107a PE (Biolegend, 1:200), and monensin (BD GolgiStop, according to manufacturer's protocol) at the beginning of the culture. Cells were harvested and stained for intracellular cytokines. 


\section{Flow cytometry}

828 Cells were harvested and passed through a 70uM nylon mesh, washed, and stained in FACS buffer $829[0.5 \%$ Bovine Serum Albumin, $0.01 \%$ sodium azide (Sigma-Aldrich) in PBS]. Cells were blocked 830 with anti-FcR (2.4G2) and then stained with amine reactive viability dyes to exclude dead cells

831 (Invitrogen). Surface antigens were stained with fluorochrome conjugated antibodies. Antibodies 832 used: anti- CD4 (GK1.5), CD19 (6D5), CD44 (IM7), CD90.1 (OX-7 and HIS51), CD95 (Fas, Jo2), 833 CD107a (1D4B), CD150 (SLAM, TC15-12F12.2), CD183 (CXCR3, CXCR3-173), CD185 834 (CXCR5, SPRCL5), CD186 (CXCR6, SA051D1), CD278 (ICOS, C398.4A), CD279 (PD1, 835 29F.1A12), CD335 (NKp46, 29A1.4), GL-7, IgD (11-26c), NK1.1 (PK136), and NKG2A/C/E (20d5). Binding to P-selectin was measured by incubating with P-selectin IgG Fusion protein (BD

837 Bioscience), washed and detected with fluorochrome conjugated secondary goat anti-human antibodies (Jackson ImmunoResearch). HA reactivity was detected using HA conjugated to FITC.

839 Following surface staining, cells were fixed with $2 \%$ paraformaldehyde (Sigma-Aldrich). For 840 intracellular staining of cytokines, cells were first surface stained then fixed with $4 \%$ 841 paraformaldehyde for 20 min, washed, and permeabilized with $0.1 \%$ saponin buffer (1\% FBS, $8420.1 \% \mathrm{NaN}_{3}$ and $0.1 \%$ saponin in PBS, (Sigma-Aldrich) for 15 mins. Subsequent staining for 843 cytokines using the following antibodies: anti-IFN $\gamma$ (XMG1.2), anti- TNF $\alpha$ (MP6-XT22). IL-21

844 was detected using IL-21RFc (R\&D systems), washed and detected with fluorochrome conjugated 845 secondary goat anti-human antibodies (Jackson ImmunoResearch). GzmB was stained 846 intracellulary directly ex vivo using anti-GzmB (GB11). For Bcl-6 staining, cells were first surface 847 stained then fixed and permeabilized using the FoxP3 fix/perm kit (eBioscience) following manufacturer's protocol and stained with anti-Bcl-6 (K112-91). Antibodies were obtained from 
849 eBioscience, Biolegend, or BD Bioscience. Stained cells were acquired on an LSRII flow

850 cytometer (BD) and analyzed using FlowJo analysis software.

\section{$851 \quad$ Statistics}

852 Unpaired, two-tailed, Students t-test was used to assess statistical significance between the means

853 of two groups, with $\mathrm{P}<0.05$ considered significant. Analysis was done using Prism (Graphpad)

854 software. Error bars in the figures represent the standard error of the mean. Significance in the 855 figures are indicated as $* P<0.05$, ** $P<0.01$ and $* * * P<0.001$. Expression levels of different 856 markers analyzed by flow cytometry are shown as MFI (Median Fluorescence Intensity) or nMFI 857 (normalized MFI). To correct for batch effects while pooling data from different experiments, we 858 normalized MFI by dividing each data point within an experiment by the average MFI of the 859 control group from that experiment. $\mathrm{nMFI}=\mathrm{MFI} /($ average MFI of the control group)

\section{Study approval}

861 Experimental animal procedures were done in accordance with UMMS Animal Care and Use 862 Committee guidelines that meet IACUC guidelines. 


\section{REFERENCES}

871

872

873

874

875

876

877

878

879

880

881

882

883

884

885

886

887

888

889

890

891

892

893

894

895

896

897

898

899

900

901

902

903

904

905

906

907

908

909

Allie, S.R., Bradley, J.E., Mudunuru, U., Schultz, M.D., Graf, B.A., Lund, F.E., and Randall, T.D. (2019). The establishment of resident memory B cells in the lung requires local antigen encounter. Nat Immunol 20, 97-108.

Au-Yeung, B.B., Zikherman, J., Mueller, J.L., Ashouri, J.F., Matloubian, M., Cheng, D.A., Chen, Y., Shokat, K.M., and Weiss, A. (2014). A sharp T-cell antigen receptor signaling threshold for Tcell proliferation. Proc Natl Acad Sci U S A 111, E3679-3688.

Barnden, M.J., Allison, J., Heath, W.R., and Carbone, F.R. (1998). Defective TCR expression in transgenic mice constructed using cDNA-based alpha- and beta-chain genes under the control of heterologous regulatory elements. Immunol Cell Biol 76, 34-40.

Baumjohann, D., Preite, S., Reboldi, A., Ronchi, F., Ansel, K.M., Lanzavecchia, A., and Sallusto, F. (2013). Persistent antigen and germinal center B cells sustain T follicular helper cell responses and phenotype. Immunity 38, 596-605.

Bautista, B.L., Devarajan, P., McKinstry, K.K., Strutt, T.M., Vong, A.M., Jones, M.C., Kuang, Y., Mott, D., and Swain, S.L. (2016). Short-Lived Antigen Recognition but Not Viral Infection at a Defined Checkpoint Programs Effector CD4 T Cells To Become Protective Memory. J Immunol 197, 3936-3949.

Beura, L.K., Wijeyesinghe, S., Thompson, E.A., Macchietto, M.G., Rosato, P.C., Pierson, M.J., Schenkel, J.M., Mitchell, J.S., Vezys, V., Fife, B.T., et al. (2018). T Cells in Nonlymphoid Tissues Give Rise to Lymph-Node-Resident Memory T Cells. Immunity 48, 327-338 e325.

Botta, D., Fuller, M.J., Marquez-Lago, T.T., Bachus, H., Bradley, J.E., Weinmann, A.S., Zajac, A.J., Randall, T.D., Lund, F.E., Leon, B., and Ballesteros-Tato, A. (2017). Dynamic regulation of $\mathrm{T}$ follicular regulatory cell responses by interleukin 2 during influenza infection. Nat Immunol 18, 1249-1260.

Brahmakshatriya, V., Kuang, Y., Devarajan, P., Xia, J., Zhang, W., Vong, A.M., and Swain, S.L. (2017). IL-6 Production by TLR-Activated APC Broadly Enhances Aged Cognate CD4 Helper and B Cell Antibody Responses In Vivo. J Immunol 198, 2819-2833.

Broadley, I., Pera, A., Morrow, G., Davies, K.A., and Kern, F. (2017). Expansions of Cytotoxic CD4(+)CD28(-) T Cells Drive Excess Cardiovascular Mortality in Rheumatoid Arthritis and Other Chronic Inflammatory Conditions and Are Triggered by CMV Infection. Front Immunol 8, 195.

Brown, D.M., Lee, S., Garcia-Hernandez Mde, L., and Swain, S.L. (2012). Multifunctional CD4 cells expressing gamma interferon and perforin mediate protection against lethal influenza virus infection. J Virol 86, 6792-6803.

Crotty, S. (2019). T Follicular Helper Cell Biology: A Decade of Discovery and Diseases. Immunity 50, 1132-1148.

Deenick, E.K., Chan, A., Ma, C.S., Gatto, D., Schwartzberg, P.L., Brink, R., and Tangye, S.G. (2010). Follicular helper $\mathrm{T}$ cell differentiation requires continuous antigen presentation that is independent of unique B cell signaling. Immunity 33, 241-253.

Deenick, E.K., Ma, C.S., Brink, R., and Tangye, S.G. (2011). Regulation of T follicular helper cell formation and function by antigen presenting cells. Curr Opin Immunol 23, 111-118. 
Devarajan, P., Bautista, B., Vong, A.M., McKinstry, K.K., Strutt, T.M., and Swain, S.L. (2016). New Insights into the Generation of CD4 Memory May Shape Future Vaccine Strategies for Influenza. Front Immunol 7, 136.

Devarajan, P., Jones, M.C., Kugler-Umana, O., Vong, A.M., Xia, J., and Swain, S.L. (2018). Pathogen Recognition by CD4 Effectors Drives Key Effector and Most Memory Cell Generation Against Respiratory Virus. Front Immunol 9, 596.

Dubey, C., and Croft, M. (1996). Accessory molecule regulation of naive CD4 T cell activation. Immunol Res 15, 114-125.

Fazilleau, N., McHeyzer-Williams, L.J., Rosen, H., and McHeyzer-Williams, M.G. (2009). The function of follicular helper T cells is regulated by the strength of $\mathrm{T}$ cell antigen receptor binding. Nat Immunol 10, 375-384.

Gensous, N., Charrier, M., Duluc, D., Contin-Bordes, C., Truchetet, M.E., Lazaro, E., Duffau, P., Blanco, P., and Richez, C. (2018). T Follicular Helper Cells in Autoimmune Disorders. Front Immunol 9, 1637.

Inoue, T., Moran, I., Shinnakasu, R., Phan, T.G., and Kurosaki, T. (2018). Generation of memory B cells and their reactivation. Immunol Rev 283, 138-149.

Jelley-Gibbs, D.M., Brown, D.M., Dibble, J.P., Haynes, L., Eaton, S.M., and Swain, S.L. (2005). Unexpected prolonged presentation of influenza antigens promotes CD4 $\mathrm{T}$ cell memory generation. J Exp Med 202, 697-706.

Juno, J.A., van Bockel, D., Kent, S.J., Kelleher, A.D., Zaunders, J.J., and Munier, C.M. (2017). Cytotoxic CD4 T Cells-Friend or Foe during Viral Infection? Front Immunol 8, 19.

Khan, T.N., Mooster, J.L., Kilgore, A.M., Osborn, J.F., and Nolz, J.C. (2016). Local antigen in nonlymphoid tissue promotes resident memory CD8+ T cell formation during viral infection. $\mathrm{J}$ Exp Med 213, 951-966.

Koutsakos, M., McWilliam, H.E.G., Aktepe, T.E., Fritzlar, S., Illing, P.T., Mifsud, N.A., Purcell, A.W., Rockman, S., Reading, P.C., Vivian, J.P., et al. (2019a). Downregulation of MHC Class I Expression by Influenza A and B Viruses. Front Immunol 10, 1158.

Koutsakos, M., Nguyen, T.H.O., and Kedzierska, K. (2019b). With a Little Help from T Follicular Helper Friends: Humoral Immunity to Influenza Vaccination. J Immunol 202, 360-367.

Krishnaswamy, J.K., Alsen, S., Yrlid, U., Eisenbarth, S.C., and Williams, A. (2018). Determination of T Follicular Helper Cell Fate by Dendritic Cells. Front Immunol 9, 2169.

Lee, J.Y., Skon, C.N., Lee, Y.J., Oh, S., Taylor, J.J., Malhotra, D., Jenkins, M.K., Rosenfeld, M.G., Hogquist, K.A., and Jameson, S.C. (2015). The transcription factor KLF2 restrains CD4(+) T follicular helper cell differentiation. Immunity 42, 252-264.

Lewis, S.M., Williams, A., and Eisenbarth, S.C. (2019). Structure and function of the immune system in the spleen. Sci Immunol 4.

Linterman, M.A., Denton, A.E., Divekar, D.P., Zvetkova, I., Kane, L., Ferreira, C., Veldhoen, M., Clare, S., Dougan, G., Espeli, M., and Smith, K.G. (2014). CD28 expression is required after T cell priming for helper $\mathrm{T}$ cell responses and protective immunity to infection. Elife 3. 
Malhotra, D., Fletcher, A.L., and Turley, S.J. (2013). Stromal and hematopoietic cells in secondary lymphoid organs: partners in immunity. Immunol Rev 251, 160-176.

Marshall, N.B., and Swain, S.L. (2011). Cytotoxic CD4 T cells in antiviral immunity. J Biomed Biotechnol 2011, 954602.

Marshall, N.B., Vong, A.M., Devarajan, P., Brauner, M.D., Kuang, Y., Nayar, R., Schutten, E.A., Castonguay, C.H., Berg, L.J., Nutt, S.L., and Swain, S.L. (2016). NKG2C/E Marks the Unique Cytotoxic CD4 T Cell Subset, ThCTL, Generated by Influenza Infection. J Immunol.

McKinstry, K.K., Strutt, T.M., Bautista, B., Zhang, W., Kuang, Y., Cooper, A.M., and Swain, S.L. (2014). Effector CD4 T-cell transition to memory requires late cognate interactions that induce autocrine IL-2. Nat Commun 5, 5377.

McKinstry, K.K., Strutt, T.M., Kuang, Y., Brown, D.M., Sell, S., Dutton, R.W., and Swain, S.L. (2012). Memory CD4+ T cells protect against influenza through multiple synergizing mechanisms. $\mathrm{J}$ Clin Invest 122, 2847-2856.

McMaster, S.R., Wein, A.N., Dunbar, P.R., Hayward, S.L., Cartwright, E.K., Denning, T.L., and Kohlmeier, J.E. (2018). Pulmonary antigen encounter regulates the establishment of tissueresident CD8 memory $\mathrm{T}$ cells in the lung airways and parenchyma. Mucosal Immunol 11, 10711078.

Melssen, M., and Slingluff, C.L., Jr. (2017). Vaccines targeting helper $\mathrm{T}$ cells for cancer immunotherapy. Curr Opin Immunol 47, 85-92.

Moran, A.E., Holzapfel, K.L., Xing, Y., Cunningham, N.R., Maltzman, J.S., Punt, J., and Hogquist, K.A. (2011). T cell receptor signal strength in Treg and iNKT cell development demonstrated by a novel fluorescent reporter mouse. J Exp Med 208, 1279-1289.

Moyer, T.J., Kato, Y., Abraham, W., Chang, J.Y.H., Kulp, D.W., Watson, N., Turner, H.L., Menis, S., Abbott, R.K., Bhiman, J.N., et al. (2020). Engineered immunogen binding to alum adjuvant enhances humoral immunity. Nat Med 26, 430-440.

Moyer, T.J., Zmolek, A.C., and Irvine, D.J. (2016). Beyond antigens and adjuvants: formulating future vaccines. J Clin Invest 126, 799-808.

Muraro, E., Merlo, A., Martorelli, D., Cangemi, M., Dalla Santa, S., Dolcetti, R., and Rosato, A. (2017). Fighting Viral Infections and Virus-Driven Tumors with Cytotoxic CD4(+) T Cells. Front Immunol 8, 197.

Phetsouphanh, C., Pillai, S., and Zaunders, J.J. (2017). Editorial: Cytotoxic CD4+ T Cells in Viral Infections. Front Immunol 8, 1729.

Schenkel, J.M., Fraser, K.A., and Masopust, D. (2014). Cutting edge: resident memory CD8 T cells occupy frontline niches in secondary lymphoid organs. J Immunol 192, 2961-2964.

Scott, B., Liblau, R., Degermann, S., Marconi, L.A., Ogata, L., Caton, A.J., McDevitt, H.O., and Lo, D. (1994). A role for non-MHC genetic polymorphism in susceptibility to spontaneous autoimmunity. Immunity 1, 73-83.

Serroukh, Y., Gu-Trantien, C., Hooshiar Kashani, B., Defrance, M., Vu Manh, T.P., Azouz, A., Detavernier, A., Hoyois, A., Das, J., Bizet, M., et al. (2018). The transcription factors Runx3 and ThPOK cross-regulate acquisition of cytotoxic function by human Th1 lymphocytes. Elife 7 . 
989 Swain, S.L., McKinstry, K.K., and Strutt, T.M. (2012). Expanding roles for CD4(+) T cells in 990 immunity to viruses. Nat Rev Immunol 12, 136-148.

991 Takamura, S., Yagi, H., Hakata, Y., Motozono, C., McMaster, S.R., Masumoto, T., Fujisawa, M., 992 Chikaishi, T., Komeda, J., Itoh, J., et al. (2016). Specific niches for lung-resident memory CD8+ $993 \mathrm{~T}$ cells at the site of tissue regeneration enable CD69-independent maintenance. J Exp Med 213, 994 3057-3073.

995 Tam, H.H., Melo, M.B., Kang, M., Pelet, J.M., Ruda, V.M., Foley, M.H., Hu, J.K., Kumari, S., 996 Crampton, J., Baldeon, A.D., et al. (2016). Sustained antigen availability during germinal center 997 initiation enhances antibody responses to vaccination. Proc Natl Acad Sci U S A 113, E6639998 E6648.

999 van de Berg, P.J., van Leeuwen, E.M., ten Berge, I.J., and van Lier, R. (2008). Cytotoxic human 1000 CD4(+) T cells. Curr Opin Immunol 20, 339-343.

1001 Vinuesa, C.G., Linterman, M.A., Yu, D., and MacLennan, I.C. (2016). Follicular Helper T Cells. 1002 Annu Rev Immunol 34, 335-368.

1003 Watts, T.H. (2010). Staying alive: T cell costimulation, CD28, and Bcl-xL. J Immunol 185, 378510043787. 